\title{
Axiomatizing Relativistic Dynamics using Formal Thought Experiments
}

\author{
Attila Molnár \\ Department of Logic, Institute of Philosophy, Eötvös Loránd \\ University Múzeum krt. 4/i, H-1088, Budapest, Hungary \\ Gergely Székely \\ Alfréd Rényi Institute of Mathematics, Hungarian Academy of \\ Sciences, Reáltanoda utca 13-15, H-1053, Budapest, Hungary
}

2014 August

\begin{abstract}
Thought experiments are widely used in informal explanations of Relativity Theories; however, they are not present explicitly in formalized versions of Relativity Theory. In this paper, we present an axiom system of Special Relativity which is able to express thought experiments formally and explicitly. Moreover, using these thought experiments, we can provide an explicit definition of relativistic mass based merely on kinematical concepts and thought experiments on collisions. Using this definition, we can geometrically prove the Mass Increase Formula $m_{0}=m \cdot \sqrt{1-v^{2}}$ in a natural way, without postulates of conservation of mass and momentum.
\end{abstract}

Keywords: First-order Modal Logic; Relativistic Dynamics; Thought Experiments; Definition of Mass

\section{Introduction}

David Hilbert's still open 6th problem is about to provide a foundation of physics similar to that of mathematics. The search for this foundation means to find suitable formal axiomatic systems in which we can prove the formal counterparts of predictions in physics.

Why is Hilbert's 6th problem still important? The role of basic assumptions and basic concepts in physics is at least as fundamental as in Mathematics. Therefore, it is essential to have a clear and well-structured understanding of these concepts and assumptions.

As part of this project, we would like to support predictions of physics with precise proofs. This fact also motivates us to use mathematical logic because it is currently the best framework in which we can provide the most precise proofs - since mathematical logic is exactly the discipline where it is clear what a proof is. 
Using the formal language of mathematical logic, we can clarify the tacit assumptions and opaque notions, as well as provide precise proofs for the predictions of physics.

Another advantage of using mathematical logic is the powerful device of model theory: using these tools we are not only able to decide whether an argument is correct or not, but to discover the exact boundaries of our theories. For example, we can prove that if a statement is unprovable.

Here come the methods of reverse mathematics into the picture. Using model theoretical tools we are able to examine the exact dependencies of the axioms, what is more: we can find more and more fundamental, sufficient conditions to prove an important statement. For example, [4] showed that the Mass Increase Theorem can be proved from conservation of the centerline of mass without using the conservation of mass and linear momentum. ${ }^{1}$ This means also that the Mass Increase Theorem is true even in those models where the conservation of mass or linear momentum fails (but the conservation of centerline of mass is still valid).

This reverse mathematical perspective will also be important in the present paper: we base our dynamics on an even more general foundation than what was used in [4].

At the very beginning of such a foundation, we have to choose $a$ mathematical logic. And we have to choose wisely: not all of them are suitable for axiomatization. We have to choose one which is rich enough to formulate physics, but not too rich to obscure some basic assumptions by making them "unknowable" because it decides them at the meta level, see [1, §Why FOL?], $[31, \S 11]$. The standard choice is classical first-order logic. For example, all of $[2],[3],[7],[8],[15],[22],[28]$ choose first-order logic to axiomatize relativity theories.

However, thought experiments, which are standard and commonly used tools in the everyday practice of physics, do not fit very well in these classical framework since they seem to be based on more than one model. In section 2 , we show that transformations between classical models are good candidates to represent thought experiments. One could say, that this is not surprising at all: as real experiments change the reality, thought experiments change the models of reality. The need for this research was already articulated in $[4, \S 6]$ and $[5$, pp.6-7].

Anyhow, there is a logic capable of expressing thought experiments, and is rich and safe enough to provide axiomatic bases for relativity theories. This is the first-order logic of 'possible worlds': the first-order modal logic. This paper is not the first one connecting modal logic and relativity theories. [14], [30], [29] use modalities locally to axiomatize the causal ordering of events in Minkowski spacetimes, and [18] uses first-order modal logic to eliminate the explicit use of reference frames. We use modalities to express thought experimentation, i.e., transformations between classical models of Special Relativity, more explicitly to distinguish between axioms referring to fundamental physical laws and axioms postulating fundamental properties of thought experiments.

\footnotetext{
${ }^{1}$ Another good example is that faster than light motion of particles per se is logically independent from both relativistic kinematics [32] and relativistic dynamics [25]. For an axiomatic approach defining coordinate systems moving faster than light, see [20].
} 


\subsection{Contents and Main Results}

The main results of this paper can be summarized as follows:

- We prove standard predictions of special relativity by formal thought experiments in a natural way, very close to the informal explanation. The motivation of formal thought experiments will be presented in section 2 .

- We develop a first-order modal logic axiomatization of relativistic kinematics and dynamics in which it is possible to distinguish between actual and potential objects. This will be done in section 3 and 4 .

- We define mass explicitly using thought experiments in subsection 4.1.

- We prove the relativistic Mass Increase Formula

$$
\mathrm{m}_{0}(b)=\mathrm{m}_{k}(b) \cdot \sqrt{1-\mathrm{v}_{k}(b)^{2}} .
$$

in subsection 4.3 (Thm. 11, p.29) using thought experimentation.

\section{On the Formalization of Thought Experiments}

To explore the nature of the thought experiments present in the discourse of relativity physics, we show a typical argument about that the simultaneity of events is not absolute (i.e., observer dependent): the train and platform thought experiment.

Our main assumption about the physical reality is a simple consequence of Einstein's two original postulates [12]:

The speed of light is constant for each observer. (AxPhObs)

"Theorem" 1. Simultaneity is not absolute.

"Proof" 1. Consider a train and a train station such that the train is passing by the station with constant speed. Suppose that Alice is on the train, while Bob is standing on the station. We assume that Alice is sitting in the middle of the train according to Bob. Now we show that there could be two events simultaneous according to Bob, which are not simultaneous for Alice.

To do so, let us make a thought experiment: Imagine that two lightnings strike the two ends of the train simultaneously for Bob.

By the fact that the speed of light is constant for Bob (AxPhObs), the light of the flash in front of Alice reaches her first, and (if the train is slower than light ${ }^{2}$ ) the light from her back reaches Alice second. The physical reality is the same for both Alice and Bob; therefore, Alice also observes the light signals in different events. We can assume that Alice is sitting in the middle of the train according to her as well. ${ }^{3}$ Since the speed of light is constant also for Alice according to (AxPhObs), and the two flashes occur equidistantly with respect to her, the flash in front of her occur at a different time than the one behind according to Alice, see Fig. 1. So we proved that there could be two events simultaneous for Bob but not for Alice, so the simultaneity of events is not absolute. "Q.E.D."

\footnotetext{
${ }^{2}$ The statement "no inertial observer can go faster than light" follows from the basic assumptions we use in this proof, so we can use it. For a precise proof, see [6].

${ }^{3}$ This basic statement can be proved using the very same assumptions as we use in this proof.
} 
Figure 1: Illustration of the thought experiment showing the observerdependence of simultaneity

Bob's worldview

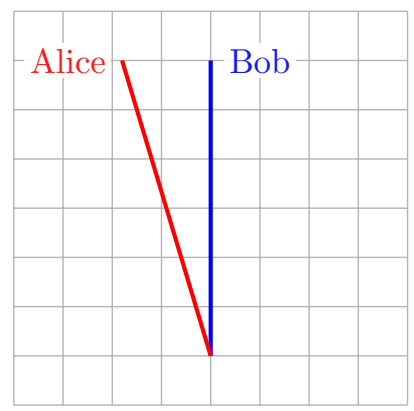

Alice's worldview

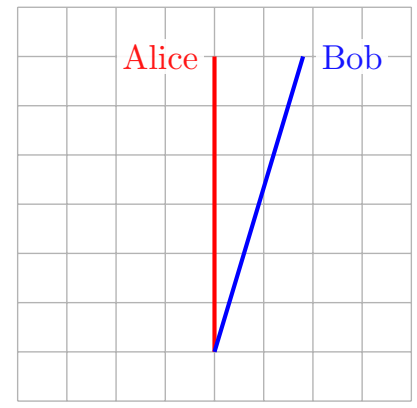

|

experiment

$\downarrow$
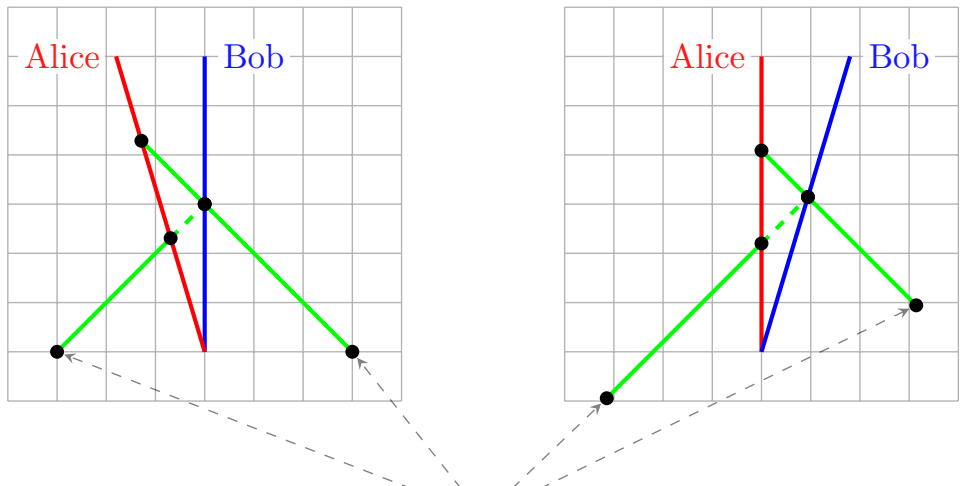

Lightning strikes

In the previous informal proof, we used a lot of natural but tacit assumptions, concerning

observations: the physical reality is the same for each observer $(A x E v)^{4}$, and observers coordinatize themselves in the origin (AxSelf), and

mathematics: since we used notions such as distance and speed, we relied on some axioms of the real numbers.

However, this proof does not work for Alice and Bob if the two flashes are not possible in the very special spacetime locations as we used in the proof: they occurred simultaneously for Bob, they were equidistant with respect to Alice, and they were oriented in the direction of the movement of Alice. This introduction of photons is a very good example for what we usually call a thought experiment. In this sense we relied on a thought experimentation axiom:

\footnotetext{
${ }^{4}$ Later we will introduce a modal version of this assumption, see AxMEv on p.15.
} 
Light Signal Sending Thought Experiments: In every spacetime location, in every direction, it is possible to send out a light signal. (AxPhExp)

Why postulating the existence of "possible" photons are legitimate? For example, because the notion of simultaneity should be independent from the actual existence of some photons, i.e., the simultaneity should be understood in terms of possible events.

But in what sense are the two flashes in the example "possible"? Logical consistency is a good starting point: the two flashes are possible because their existence does not violate the other axioms we use (e.g., AxPhObs or Einstein's more general postulates). Then the step of introduction of the two photons can be interpreted as a transformation of a model of the (classical) axioms into a very similar model of the same axioms: this model is more only in the aspect that it has two extra photons in the locations where we need them to be. So a model transformation which expands the model with two photons obeying AxPhObs is a good candidate for a formal counterpart of the light signal sending thought experiment AxPhExp. This gives the idea that physical thought experiments should be formalized as transformations of classical models.

Logic for thought experiments. Non-trivial transformations of models are always understood between different models: models before and after the transformation. However, according to classical logical semantics, the truth of a classical formula is always decided by a single model. Therefore, if we want to axiomatize at least some of these transformations, i.e., if we want to find a formula in the object language whose truth corresponds to a non-trivial modeltransformation, then such a formula (and its semantics) cannot be classical, since its truth is based on more than one model.

The solution comes from modal logic. A modal model is a set of classical models connected with a relation. This relation can represent thought experimentations, i.e., model transformations. While the thought experimentationfree (classical) formulas are evaluated in the usual way, we introduce the (modal) formulas $\nabla \varphi$ with the intended meaning of "there is a transformed model in which $\varphi$ is true" or "there is a thought experiment such that $\varphi . "$

Formally: In the classical model $w$ of the modal model $\mathfrak{M}$, the formula $\nabla \varphi$ is true iff there is a ("transformed") model $v$ such that $w R v$ and $\varphi$ is true in $v{ }^{5}$ 6

We will treat thought experiments very generously: any model transformation will count as a representation of some thought experiment. We can afford this liberty, since we will never have to use all of the model transformations or thought experiments; a selected group of model transformations is enough.

\footnotetext{
${ }^{5}$ Note that the starting idea, that thought experiments should be understood as tests for logical consistency, is fulfilled. The truth of $\nabla \varphi$ involves also classical logical consistency with the classical axioms. If $\nabla \varphi$ is true, then there is a (transformed) classical model in which $\varphi$ is true. Since the classical axioms must be true in each world of the modal model, they are also true in the transformed model. This means that $\varphi$ is consistent.

${ }^{6}$ The modal operator $\diamond$ is designed to handle a restricted quantification over possible worlds in the metalanguage. Even though all the notions, such as interpretations and models are already present in classical semantics, using modal logic is not superfluous because the purpose of modalities is not only to quantify over models, but to do this from the object language. This fact makes it possible to axiomatize thought experiments or model transformations, which is a central goal of this paper.
} 
Therefore, our aim is not to find or draw the borders of conceivability, only to find a small group of model transformations such that

(1) they deserved to be called thought experiments, and

(2) they are sufficient for a natural axiomatization of relativistic dynamics.

The job of our modal axioms is exactly to define implicitly this group. For example, if we call the members of this group relativistic dynamics thought experiments, or RDTE-s, then AxPhExp says that for all observers and all of their light-like separated coordinate points there are RDTEs such that a photon crosses the light-like separated coordinate points of the observer in the transformed model. ${ }^{7}$

\section{Kinematics}

\subsection{Language}

Since we will reduce the notion of mass to kinematical notions, the language and models of Dynamics will be very similar to that of Kinematics'. The only difference will be the presence of an individual constant naming the mass-standard body to determine the standard unit of mass. Therefore, we discuss the language and models of Dynamics now.

Our main predicate is about coordinatization:

$$
\begin{gathered}
\mathrm{W}(k, b, t, x, y, z) \text { : "Observer } k \text { coordinatizes body } b \\
\text { at the spacetime location }(t, x, y, z) . "
\end{gathered}
$$

We will use mathematical variables $x, y, z, t, x_{1}, \ldots$ to denote numbers, e.g., coordinates, and physical variables $b, c, d, k, l, h, m, \ldots$ to denote bodies and observers. We will assume that every observer is a body but not the other way around. For this differentiation, we introduce a predicate for inertial observers:

$$
\operatorname{IOb}(k): \text { " } k \text { is an inertial observer," where } k \text { is a physical term. }
$$

Since we stay in Special Relativity, in the rest of this paper we omit the expression "inertial."

Light signals play an important role in Relativity Theories; so we introduce a primitive predicate for them as well:

$$
\mathrm{Ph}(k): \text { " } k \text { is a light signal," where } k \text { is a physical term. }
$$

Our only non-variable primitive physical term is the individual constant $\varepsilon$ which represents the mass-standard, and as such will play a central role in Dynamics in section 4 .

In the case of mathematics, we use the usual basic operations,$+ \cdot$ and the ordering $\leq$.

\footnotetext{
${ }^{7}$ Maybe it would be more accurate at the introduction of modal axioms to include the precise metalinguistic characterization of the expressed model transformations, but we will set aside from this. Besides of its technical nature, it does not affect the success of axiomatization of special relativistic dynamics.
} 
To form complex formulas, we use the usual classical connectives $\neg, \wedge, \vee$, $\rightarrow, \forall, \exists$ to express "not", "and", "or", "if-then", "for all", "there exists", respectively. We use the following abbreviations to simplify our formulas:

$$
\begin{gathered}
(\exists b \in \varphi) \psi \stackrel{\text { def. }}{\Longleftrightarrow} \exists b(\varphi(b) \wedge \psi), \quad(\forall b \in \varphi) \psi \stackrel{\text { def. }}{\Longleftrightarrow} \forall b(\varphi(b) \rightarrow \psi), \\
(\exists x<\tau) \psi \stackrel{\text { def. }}{\Longleftrightarrow} \exists x(x<\tau \wedge \psi), \quad(\forall x<\tau) \psi \stackrel{\text { def. }}{\Longleftrightarrow} \forall x(x<\tau \rightarrow \psi) .
\end{gathered}
$$

For the same reason, we refer to $n$-tuples using the vector notation:

$$
\forall \bar{x} \varphi(\bar{x}) \stackrel{\text { def. }}{\Longleftrightarrow} \forall x_{1}, \ldots, x_{n} \varphi\left(x_{1}, \ldots, x_{n}\right) .
$$

Our only non-classical connective is the modal operator $\diamond$ with the intended meaning that "there is a thought experiment according to which..." or "the actual model can be transformed in a way such that...". We define the dual operator $\square \varphi \stackrel{\text { def. }}{=} \neg \diamond \neg \varphi$; hence $\square \varphi$ is true iff " $\varphi$ is invariant under model transformations/thought experiments." Therefore, an axiom of the form $\square \varphi$ means that "we use only those thought experiments according to which $\varphi$ is invariant."

\subsection{Semantics}

A model for MSpecRel:

$$
\mathfrak{M}=\left\langle\mathfrak{Q}, \mathfrak{P}, \mathrm{W}^{\mathfrak{M}}\right\rangle \quad \text { where } \quad \begin{aligned}
& \mathfrak{Q}=\left\langle Q,+\mathfrak{M}, \stackrel{\mathfrak{M}}{\mathfrak{P}}=\left\langle S, \leq^{\mathfrak{M}}\right\rangle, D, \mathrm{IOb}^{\mathfrak{M}}, \mathrm{Ph}^{\mathfrak{M}}, \varepsilon^{\mathfrak{M}}\right\rangle \\
& \mathfrak{P}
\end{aligned}
$$

Here $\mathfrak{Q}$ is the mathematical and classical (Tarskian) part of the model:

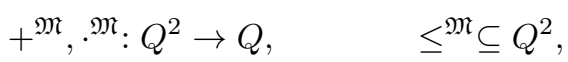

and $\mathfrak{P}$ is the physical and modal part of the model. The set $S$ is the set of possible worlds, which is a nonempty set used for naming the classical first-order models.

$R$ is a reflexive binary relation on $S$ called the alternative-relation. The purpose of this relation is to select those possible worlds which can be reached from the actual world by thought experiments. The precise calibration of this relation will be done by axioms containing modal operators $\square$ and $\diamond$.

$D$ is a function assigning to each $w \in S$ a (possibly empty) set $D_{w}$. These sets are considered to be the domains of physical quantification, or simply the set of existing or "actual" physical objects in the world $w$. The possible objects are the objects that are "actual-in-some-possible-world":

$$
U \stackrel{\text { def. }}{=} \bigcup_{w \in S} D_{w}
$$

$\mathrm{IOb}^{\mathfrak{M}}$ and $\mathrm{Ph}^{\mathfrak{M}}$ are modal predicates for observers and photons. Since the sets of observers and photons can vary in different worlds, the modal predicates are functions assigning subsets of $U$ to each world $w$ :

$$
\mathrm{IOb}^{\mathfrak{M}}, \mathrm{Ph}^{\mathfrak{M}}: S \rightarrow \mathcal{P}(U) .
$$

Function $\varepsilon^{\mathfrak{M}}$ assigns a possible object, the one and only (and not necessarily existing) mass-standard for each $w \in S$ in a way that the denotation of $\varepsilon$ cannot vary between $R$ connected worlds (i.e., it is a so-called rigid designator):

$$
\varepsilon^{\mathfrak{M}}: S \rightarrow U \text { and } w R v \Rightarrow \varepsilon_{w}^{\mathfrak{M}}=\varepsilon_{v}^{\mathfrak{M}} .
$$


Finally $\mathrm{W}^{\mathfrak{M}}$ is the hybrid modal and classical predicate for coordinatization. This is also a function, since the worldviews can vary from world to world:

$$
\mathrm{W}_{w}^{\mathfrak{M}} \subseteq D_{w}^{2} \times Q^{4}
$$

Assignments. Let $\sigma_{Q}$ be an assignment of the classical part of the model in the classical sense, i.e., a function assigning the elements of $Q$ to the mathematical variables. In the case of the physical and modal parts, let an assignment $\sigma_{U}$ map possible individuals (i.e., elements of $U$ ) to the physical variables. Then a two-sorted assignment for a model of MSpecRel:

$$
\sigma(x) \stackrel{\text { def. }}{=} \begin{cases}\sigma_{Q}(x) & \text { if } x \text { is a mathematical variable } \\ \sigma_{U}(x) & \text { if } x \text { is a physical variable }\end{cases}
$$

We define the $x$-variant assignments in the usual way:

$$
\sigma \stackrel{x}{\equiv} \tau \stackrel{\text { def. }}{\Longleftrightarrow} \text { for all } y \neq x: \sigma(y)=\tau(y) \text {. }
$$

Terms. The denotation of terms are defined in the usual way:

$$
t^{\mathfrak{M}, w, \sigma} \stackrel{\text { def. }}{=} \begin{cases}\sigma(t) & \text { if } t \text { is a variable, } \\ f_{i}^{\mathfrak{M}, w, \sigma}\left(t_{1}^{\mathfrak{M}, w, \sigma}, \ldots, t_{n}^{\mathfrak{M}, w, \sigma}\right) & \text { if } t=f_{i}\left(t_{1}, \ldots, t_{n}\right) .\end{cases}
$$

Truth. To define truth, we introduce the following notation:

$$
\mathfrak{M}, w \models \varphi[\sigma] \text {. }
$$

We read this in the following way: $\varphi$ is true in the world $w$ of the modal model $\mathfrak{M}$ according to an assignment $\sigma$. The precise definition is given by recursion: The truth of the atomic sentences made by $=$ and $\mathrm{W}$ :

$$
\begin{array}{lll}
\mathfrak{M}, w=\mathrm{W}(k, b, \bar{x})[\sigma] & \stackrel{\text { def. }}{\Longleftrightarrow} & \left\langle k^{\mathfrak{M}}, b^{\mathfrak{M}}, \bar{x}^{\mathfrak{M}}\right\rangle \in \mathrm{W}_{w}^{\mathfrak{M}}, \\
\mathfrak{M}, w=t_{1}=t_{2}[\sigma] \quad & \Longleftrightarrow & t_{1}^{\mathfrak{M}}=t_{2}^{\mathfrak{M}} .
\end{array}
$$

The truth of the other atomic formulas is defined similarly. The truth of formulas connected by $\wedge, \vee, \rightarrow$ and $\leftrightarrow$ are defined in the usual way; however, the truth of the quantified and modalized formulas are special:

$$
\begin{aligned}
\mathfrak{M}, w \models \exists x \varphi[\sigma] \stackrel{\text { def. }}{\Longleftrightarrow}\left\{\begin{array}{l}
\text { there exists a } \tau \equiv{ }^{x} \sigma \text { such that } \\
\mathfrak{M}, w \models \varphi[\tau],
\end{array}\right. \\
\mathfrak{M}, w \models \exists b \varphi[\sigma] \stackrel{\text { def. }}{\Longleftrightarrow}\left\{\begin{array}{l}
\text { there exists a } \tau \equiv{ }^{b} \sigma \text { such that } \\
\tau(b) \in D_{w} \text { and } \mathfrak{M}, w \models \varphi[\tau],
\end{array}\right. \\
\mathfrak{M}, w \models \nabla \varphi[\sigma] \stackrel{\text { def. }}{\Longleftrightarrow}\left\{\begin{array}{l}
\text { there exists a } w^{\prime} \in S \text { such that } \\
w R w^{\prime} \text { and } \mathfrak{M}, w^{\prime} \models \varphi[\sigma] .
\end{array}\right.
\end{aligned}
$$

Note that in the case of the physical sort, we quantify over $D_{w}$, i.e., over the actually existing bodies. The possible bodies are only accesible using modalities, such as $\diamond \exists b, \square \diamond \diamond \forall b$, etc.

A formula is said to be true in a model, $\mathfrak{M} \models \varphi$ iff it is true in all of its worlds according to any assignment. 
Figure 2: An example for a first-order modal model of our language

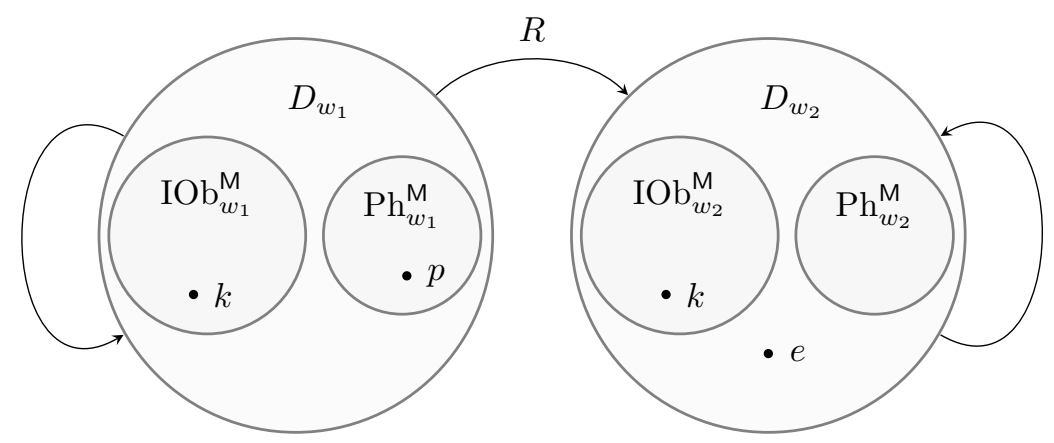

The worlds we use are not ordinary classical models, because the classical axiom schema of universal instantiation $(\forall b \varphi(b) \rightarrow \varphi(t / b))$ is false in them. To show this, we give a simple example: Consider the following model illustrated on Fig. 2:

$$
\left\langle\mathbb{R}, S, R, D, \mathrm{IOb}^{\mathfrak{M}}, \mathrm{Ph}^{\mathfrak{M}}, \varepsilon^{\mathfrak{M}}, \mathrm{W}^{\mathfrak{M}}\right\rangle
$$

- $\mathbb{R}$ is the field of real numbers.

- There are only two worlds $w_{1}$ and $w_{2}$, i.e., $S=\left\{w_{1}, w_{2}\right\}$, such that $w_{2}$ is a transformed version of $w_{1}$, and both worlds are transformed versions of themselves:

$$
R=\left\{\left\langle w_{1}, w_{2}\right\rangle,\left\langle w_{1}, w_{1}\right\rangle,\left\langle w_{2}, w_{2}\right\rangle\right\}
$$

- In both worlds, there exist only two entities: $D_{w_{1}}=\{k, p\}, D_{w_{2}}=\{k, e\}$. So the possible entities are $U=\{k, p, e\}$.

- $k$ is an observer in both worlds, $\mathrm{IOb}_{w_{1}}=\mathrm{IOb}_{w_{2}}=\{k\}, p$ is a photon in $w_{1}, \mathrm{Ph}_{w_{1}}=\{p\}, \mathrm{Ph}_{w_{2}}=\varnothing, e$ is the mass-standard of $w_{1}$ and $w_{2}$, i.e., $e=\varepsilon_{w_{1}}^{\mathfrak{M}}=\varepsilon_{w_{2}}^{\mathfrak{M}}$ (they cannot differ, since $\left.w_{1} R w_{2}\right)$.

- $k$ sees itself in the origin in both worlds, $k$ coordinatize $p$ moving from 0 in the direction of its $x$-axis in the world $w_{1}, e$ is stationary for $k$ in $w_{2}$.

$$
\begin{aligned}
& \mathrm{W}_{w_{1}}^{\mathfrak{M}}=\left\{\langle a, b, t, x, y, z\rangle: k=a \text { and } \begin{array}{l}
\text { if } b=k \text { then } x=y=z=0, \\
\text { if } b=p \text { then } t=x, y=z=0,
\end{array}\right\} \\
& \mathrm{W}_{w_{2}}^{\mathfrak{M}}=\left\{\langle a, b, t, x, y, z\rangle: k=a \text { and } \begin{array}{l}
\text { if } b=k \text { then } x=y=z=0, \\
\text { if } b=e \text { then } x=2, y=z=0,
\end{array}\right\}
\end{aligned}
$$

Let us now consider formula $(\exists b) b=\varepsilon$ expressing that $\varepsilon$ exists. For expressing existence this way, we use the following abbreviation:

$$
\mathrm{E}(c) \stackrel{\text { def. }}{\Longleftrightarrow}(\exists b) b=c .
$$

Now $\mathrm{E}(\varepsilon)$ is true in $w_{2}$ but not in $w_{1}$, since $\varepsilon_{w_{1}}^{\mathfrak{M}}=e \notin D_{w_{1}}$. However, the formula $\forall b \mathrm{E}(b)$ is true in $w_{1}$, since $\mathrm{E}(b)$ and $\mathrm{E}(p)$ are true, but since for the 
truth of $\forall$-statements we examine only the elements of $D_{w_{1}}$, the falsity of $\mathrm{E}(\varepsilon)$ does not count. This means that in our models the classical axiom schema of universal instantiation,

$$
\forall b \varphi(b) \rightarrow \varphi(t / b)
$$

fails. Therefore, we do the standard abstraction present in the first-order modal literature (see [11], [16]): we replace this by the actual instantiation schema:

$$
\mathrm{E}(t) \rightarrow(\forall b \varphi(b) \rightarrow \varphi(t / b)) .
$$

\subsection{Logical axioms}

The logical axioms are

- the usual axioms and derivation rules of classical propositional logic.

- the usual axioms and derivation rules of classical first-order logic for mathematics.

- the usual axioms and derivation rules of classical first-order logic for physics, except the law (UI). We use (AI) instead.

- [13] showed that this system still not proves that the quantifiers of the same sort commute. We postulate these commutativities and we let commutate the quantifications of different sorts too:

$$
\forall b \forall c \varphi \leftrightarrow \forall c \forall b \varphi \quad \forall b \forall x \varphi \leftrightarrow \forall x \forall b \varphi .
$$

- the usual axioms of identity for both sorts, and a new modal axiom expressing that non-identity is invariant under thought experiments. During the axiomatization of special relativity we do not use such a radical thought experiment which could merge two objects into one single object.

$$
\begin{aligned}
t=t, \quad t=s \rightarrow(\varphi(t / x) & \rightarrow \varphi(s / x)), \quad t=s \rightarrow(\varphi(t / b) \rightarrow \varphi(s / b)), \\
t & \neq s \rightarrow \square(s \neq t) .
\end{aligned}
$$

- the axiom and the derivation rule of the most general normal modal propositional logic $\mathbf{K}$ :

$$
\square(\varphi \rightarrow \psi) \rightarrow(\square \varphi \rightarrow \square \psi), \quad \frac{\varphi}{\square \varphi} .
$$

For us, these express that the modal logical tautologies are invariant under thought experiments, and that invariance under thought experiments is closed to modus ponens.

- For simplicity, we assume that every world counts as a transformed version of itself, i.e., $R$ is reflexive. ${ }^{8}$ The standard way ${ }^{9}$ to axiomatize reflexivity is to take the propositional axiom

$$
\varphi \rightarrow \diamond \varphi
$$

\footnotetext{
${ }^{8}$ However, this assumption can be evaded by replacing $\square \varphi$ and $\diamond \varphi$ with $\varphi \wedge \square \varphi$ and $\varphi \vee \diamond \varphi$ in all our axioms.

${ }^{9}$ For the standard line of thought, see [9, Def. 3.3, Example 3.6].
} 
This proof system is strongly complete with respect to the semantics we use, see [11, Thm. 2.9 (i), p. 1502.]. ${ }^{10}$

\subsection{Mathematical Axioms}

For the mathematical part, we use the theory of Euclidean fields.

Axiom 1 (Axioms of Euclidean Fields).

AxEField The mathematical part of the model is a Euclidean field, i.e., an ordered field ${ }^{11}$ in which every positive number has a square root. ${ }^{12}$

\subsection{Axiom for characterizing the Framework}

Here we specify some minimal requirements on the thought experimentation we will use.

Axiom 2 (Axioms of Modal Framework).

AxMFrame Mathematics is invariant under thought experiments, and every (existing) observer remains an existing observer, i.e., the observers and their ability to coordinatize cannot vanish after a thought experiment:

$$
\begin{aligned}
& (\forall k \in \mathrm{IOb}) \square(\mathrm{E}(k) \wedge \mathrm{IOb}(k)), \\
& (\forall x, y, z) \quad x+y=z \quad \leftrightarrow \quad \square x+y=z, \\
& (\forall x, y, z) \quad x \cdot y=z \quad \leftrightarrow \quad \square x \cdot y=z, \\
& (\forall x, y) \quad x \leq y \quad \leftrightarrow \quad \square x \leq y, \\
& (\forall x, y) \quad x=y \quad \leftrightarrow \quad \square x=y .
\end{aligned}
$$

Note that AxMFrame allows an object to be an observer in a world $w$ and a non-observer in an other world $w^{\prime}$. This axiom ensures only that $w^{\prime}$ cannot be a transformed version of $w$, i.e., the relation $R$ cannot connect them in this order.

The postulates about atomic statements of the mathematical sort implies that $\mu \leftrightarrow \square \mu$ whenever $\mu$ is a "purely" mathematical formula. Practically, these axioms say that we do not consider thought experimentations according to which $2+2$ can be 5 .

\subsection{Physical axioms}

In our first physical axiom, we use the following notations:

$$
\bar{x} \in \text { wline }_{k}(b) \stackrel{\text { def. }}{\Longleftrightarrow} \mathrm{W}(k, b, \bar{x}),
$$

\footnotetext{
$10[11]$ proved strong completeness for only one-sorted modal languages, but our language can be interpreted into it in the usual way, i.e., we introduce a $D$ and a $Q$ predicate to distinguish the sorts. To construct one-sorted models for our system, we only have to stipulate that the mathematical parts of the $R$-connected worlds are the same, i.e., they are invariant under $R$.

$[16,5.6]$ is also a recent source of a strong completeness theorem, which is too general for our present purpose (it is designed to incorporate even nonrigid designator terms); however, using that approach would probably be more elegant especially for readers preferring algebraic approaches.

${ }^{11}$ For the axioms of ordered fields, see e.g., [10, p.41].

${ }^{12}$ That is, $(\forall x>0)(\exists y) x=y^{2}$.
} 


$$
\begin{gathered}
\bar{x}_{t} \stackrel{\text { def. }}{=} x_{1}, \quad \bar{x}_{s} \stackrel{\text { def. }}{=}\left(x_{2}, x_{3}, x_{4}\right), \\
\operatorname{Time}(\bar{x}, \bar{y}) \stackrel{\text { def. }}{=}\left|\bar{x}_{t}-\bar{y}_{t}\right|, \quad \operatorname{Space}(\bar{x}, \bar{y}) \stackrel{\text { def. }}{=}\left|\bar{x}_{s}-\bar{y}_{s}\right| .
\end{gathered}
$$

Axiom 3 (Axiom of Observation of Light Signals.).

AxPhObs Every observer sees the worldlines of photons as of slope 1. See Fig. 3:

$$
(\forall k \in \mathrm{IOb})(\forall \bar{x}, \bar{y})\left((\exists p \in \mathrm{Ph}) \bar{x}, \bar{y} \in \operatorname{wline}_{k}(p) \rightarrow \frac{\operatorname{Space}(\bar{x}, \bar{y})}{\operatorname{Time}(\bar{x}, \bar{y})}=1\right) .
$$

Figure 3: Axiom of Observation of Light Signals
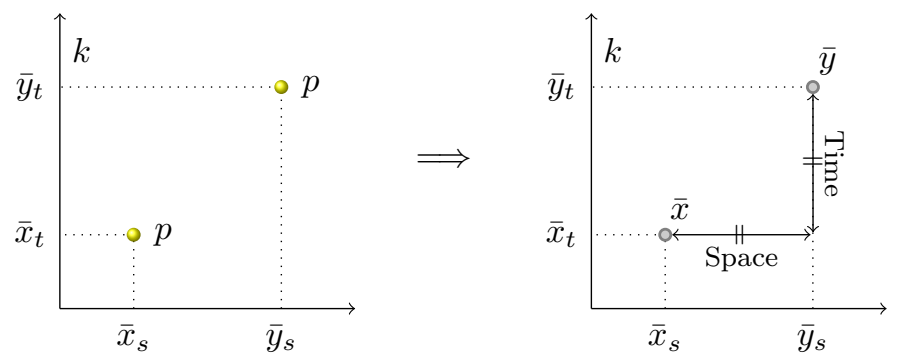

Axiom 4 (Axiom of Light Signal Sending).

AxPhExp Every observer can send a photon through coordinate points of slope 1. See Fig. 4:

$$
(\forall k \in \mathrm{IOb})(\forall \bar{x}, \bar{y})\left(\frac{\operatorname{Space}(\bar{x}, \bar{y})}{\operatorname{Time}(\bar{x}, \bar{y})}=1 \rightarrow \diamond(\exists p \in \mathrm{Ph}) \bar{x}, \bar{y} \in \text { wline }_{k}(p)\right) .
$$

This axiom practically says that if there are two spacetime locations where, according to AxPhObs, there could be a photon, (i.e., their slope is 1) then there is a thought experiment which transforms the actual world into a world in which there is a photon crossing through these spacetime locations. That was the axiom we used in the example in section 2.

The most important message of the special theory of relativity is that relatively moving observers coordinatize the world differently even with respect to time and simultaneity. So the most interesting relation of this theory must be the relation which connects the corresponding coordinate points of different observers, because if we want to say something about relativistic effects, such as time dilation, length contraction, etc., we have to compare different observers' corresponding coordinates. The usual way to achieve this is to introduce the notion of events. Intuitively an event is a meeting, an encountering, a collision etc. which itself is observer-independent. What is observer-dependent, is the spacetime location of these events in the observers' coordinate-systems. We can introduce an observer-dependent formal counterpart for the notion of event: 


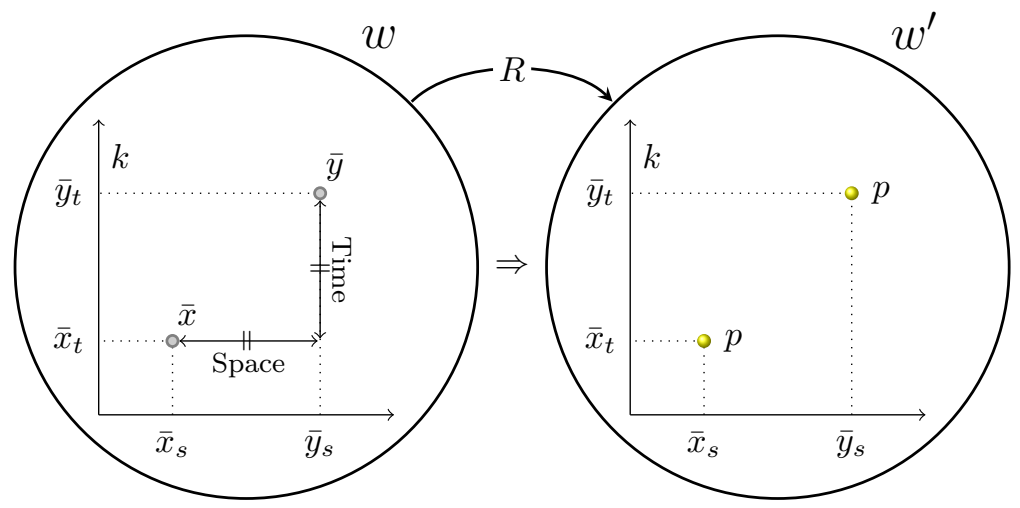

Definition 1. An event at a coordinate point $\bar{x}$ according to $k$ in a world $w$ is the set of existing (actual) bodies occurring there: ${ }^{13}$

$$
\mathrm{ev}_{k}(\bar{x}) \stackrel{\text { def. }}{=}\{b \in \mathrm{E}: \mathrm{W}(k, b, \bar{x})\} .
$$

Let $w$ and $w^{\prime}$, respectively, be the worlds before and after the thought experiment in the story of Alice and Bob in section 2. Then the possible values of $\operatorname{ev}_{\text {Alice }}(\bar{x})$ and $\operatorname{ev}_{\mathrm{Bob}}(\bar{x})$ are

$\varnothing,\{$ Alice $\},\{$ Bob $\},\{$ Alice, Bob $\}$ in $w$, and

$\varnothing,\{$ Alice $\},\{$ Bob $\},\{$ Alice, Bob $\},\left\{p_{1}\right\},\left\{p_{2}\right\},\left\{\right.$ Alice, $\left.p_{1}\right\},\left\{\right.$ Alice, $\left.p_{2}\right\}$ in $w^{\prime}$.

Let us note that Alice and Bob usually coordinatize these events in several different spacetime locations. However, they coordinatize the same events, i.e., there is no event which is available for only one of them. ${ }^{14}$

This is not the usual concept of events. In the literature, the events are interpreted as possible events, but in our case, the events are actual events since they are sets of actual bodies. Of course, there is a good reason behind the use possible events. Since different possible events correspond to different coordinate points of an observer, and every possible event is coordinatized by every observer, possible events yield the bridge between the different worldviews of observers; two coordinate points of different observers correspond to each other iff they are coordinates of the same possible event. This correspondence yields the so-called worldview transformation, i.e., the transformation which connects those points of two coordinate systems, that correspond to each other. Without the worldview transformation, in this framework, it seems to be hopeless to articulate even the most basic relativistic effects. So the question is: Can we build this bridge?

\footnotetext{
${ }^{13}$ Note that the expression on the right side of the equivalence comes from the metalanguage. We can, however, pull this definition back the object language in the following way: $b \in$ $\mathrm{ev}_{k}(\bar{x}) \stackrel{\text { def. }}{\Longleftrightarrow} \mathrm{W}(k, b, \bar{x})$.

${ }^{14}$ This assumption is axiom AxEv (the axiom of events) in the classical approach, see e.g., [6, p.638.]. Here we will use its modal version, see AxMEv on p. 15.
} 
The answer is yes, but instead of using possible events, we will use actual events and the act of the differentiating between possible events, since although the possible events are hardly expressible, the act of differentiating between them is easily expressible. ${ }^{15}$ For example, let us assume that Alice and Bob meet with each other at the coordinate point $(0,0,0,0)$ according to both of them. In the worldview of Alice, the coordinates $(0,3,0,0)$ and $(0,2,0,0)$ refer to the same actual event $\varnothing$ in the world $w$. However, we know that they refer to different possible events, even if we can not express the set of possible bodies occurring in these coordinates. The reason is that (using AxPhExp) we executed a thought experiment which produced a photon $p_{2}$ which occurred in the new actual event ev Alice $(0,3,0,0)$ but not in evalice $(0,2,0,0)$. What is more, by AxPhObs, there are no relativistic dynamics thought experiments placing $p$ in actual events at $(0,3,0,0)$ and $(0,2,0,0)$ in any world. So, however, the possible events are inexpressible in our framework, we can define the bridge between the different worldviews of observers.

Definition 2 (Worldview transformation). We say that $k$ sees at $\bar{x}$ what $h$ sees at $\bar{y}$ iff in all transformed worlds the event in $\bar{x}$ for $k$ is the same as the event in $\bar{y}$ for $h$. In other words, $k$ sees at $\bar{x}$ what $h$ sees at $\bar{y}$ iff it is impossible to tell apart these two events by thought experiments:

$$
\mathrm{w}_{k h}(\bar{x}, \bar{y}) \stackrel{\text { def. }}{\Longleftrightarrow} \square \forall b(\mathrm{~W}(k, b, \bar{x}) \leftrightarrow \mathrm{W}(h, b, \bar{y})),
$$

or, using the notation introduced in Def. 1,

$$
\mathrm{w}_{k h}(\bar{x}, \bar{y}) \stackrel{\text { def. }}{\Longleftrightarrow} \square \operatorname{ev}_{k}(\bar{x})=\operatorname{ev}_{h}(\bar{y}) .
$$

Prop. 1 shows that AxPhExp provides enough thought experiments to prove that worldview transformations give a one-to-one correspondence between coordinate points.

Proposition 1. Worldview transformations are injective functions.

$\{$ AxEField, AxMFrame, AxPhExp, AxPhObs $\} \vdash$

$$
(\forall k, h \in \mathrm{IOb})(\forall \bar{x}, \bar{y}, \bar{z})\left(\begin{array}{c}
{\left[\left(\mathrm{w}_{k h}(\bar{x}, \bar{y}) \wedge \mathrm{w}_{k h}(\bar{x}, \bar{z})\right) \rightarrow \bar{y}=\bar{z}\right] \wedge} \\
\wedge\left[\left(\mathrm{w}_{k h}(\bar{y}, \bar{x}) \wedge \mathrm{w}_{k h}(\bar{z}, \bar{x})\right) \rightarrow \bar{y}=\bar{z}\right]
\end{array}\right)
$$

Proof. By the definition of worldview transformation, $\mathrm{w}_{k h}(\bar{x}, \bar{y})=\mathrm{w}_{h k}(\bar{y}, \bar{x})$. Therefore, $\mathrm{w}_{k h}$ is injective iff $\mathrm{w}_{h k}$ is a function. So by the symmetry of $h$ and $k$ in the statement, it is enough to prove that $\mathrm{w}_{k h}$ is a function. To do so, let us assume towards contradiction that $\mathrm{w}_{k h}(\bar{x}, \bar{y}), \mathrm{w}_{k h}(\bar{x}, \bar{z})$, but $\bar{y} \neq \bar{z}$ in a world $w$. In this case, by AxPhExp and AxPhObs, $h$ could send out a light signal from $\bar{y}$ in such a direction that it avoids $\bar{z}$, i.e., there is a possible world $w^{\prime}$, such that $w R w^{\prime}$, and in $w^{\prime}$ there is a photon $p$ such that

$$
p \in \operatorname{ev}_{h}(\bar{y}) \quad \text { but } \quad p \notin \operatorname{ev}_{h}(\bar{z}) \text {. }
$$

\footnotetext{
${ }^{15}$ The possible events are accessible in the metalanguage in a straightforward way: they are sets of possible bodies, i.e., sets of elements of $U$, occurring at a coordinate point of some observer. In the classical approach of SpecRel, we use this definition, see [6, p.637.]. However, in the modal approach, we do not have access to $U$, only to a $D_{w}$ since we can quantify over only the elements of $D_{w}$, i.e., over actual bodies.
} 
However, since it is true in $w$ that

$$
\begin{aligned}
\mathrm{w}_{k h}(\bar{x}, \bar{y}) & \Longleftrightarrow \square \mathrm{ev}_{k}(\bar{x})=\mathrm{ev}_{h}(\bar{y}), \\
\mathrm{w}_{k h}(\bar{x}, \bar{z}) & \Longleftrightarrow \square \operatorname{ev}_{k}(\bar{x})=\operatorname{ev}_{h}(\bar{z}),
\end{aligned}
$$

by the definition of $\square$, it is true also in $w^{\prime}$ that

$$
\operatorname{ev}_{k}(\bar{x})=\operatorname{ev}_{h}(\bar{y}) \text { and } \operatorname{ev}_{k}(\bar{x})=\operatorname{ev}_{h}(\bar{z}), \quad \text { hence } \quad \operatorname{ev}_{h}(\bar{y})=\operatorname{ev}_{h}(\bar{z}) .
$$

This contradicts (2), which proves that $\mathrm{w}_{k h}$ is a function.

By Prop. 1, we can use the following notation for worldview transformations:

$$
\mathrm{w}_{k h}(\bar{x})=\bar{y} \stackrel{\text { def. }}{\Longleftrightarrow} \mathrm{w}_{k h}(\bar{x}, \bar{y}) .
$$

So far we have not assumed that there is at least one corresponding coordinate point in the worldviews of observers. That is, in some sense, we have not assumed that observers coordinatize the same physical reality. This is an important statement; so we take it as an axiom.

Axiom 5 (Axiom of Events).

AxMEv The possible events are the same for every observer, i.e., there is no possible world in which there is an actual event for an observer, which is not observed by every other observers:

$$
(\forall k, h \in \operatorname{IOb})(\forall \bar{x})(\exists \bar{y}) \quad \mathrm{w}_{k h}(\bar{x})=\bar{y} .
$$

Proposition 2. If we assume AxMEv, AxPhObs and AxPhExp, then worldview transformations are bijections from $Q^{4}$ to $Q^{4}$.

Now we introduce two more axioms to standardize coordinatizations:

Axiom 6 (Axiom of Self-Coordinatization).

AxSelf Every observer coordinatizes itself stationary in the origin:

$$
(\forall k \in \mathrm{IOb})\left(\forall \bar{x} \in \text { wline }_{k}(k)\right) \quad \bar{x}_{s}=\overline{0} .
$$

Axiom 7 (Axiom of Symmetry).

AxMSym All observers use the same system of measurements:

$$
\begin{aligned}
&(\forall k, h \in \mathrm{IOb})\left(\forall \bar{x}, \bar{x}^{\prime}, \bar{y}, \bar{y}^{\prime}\right)\left(\operatorname{Time}(\bar{x}, \bar{y})=0 \wedge \operatorname{Time}\left(\bar{x}^{\prime}, \bar{y}^{\prime}\right)=0 \wedge\right. \\
&\left.\wedge \mathrm{w}_{k h}(\bar{x})=\bar{x}^{\prime} \wedge \mathrm{w}_{k h}(\bar{y})=\bar{y}^{\prime}\right) \rightarrow \operatorname{Space}(\bar{x}, \bar{y})=\operatorname{Space}\left(\bar{x}^{\prime}, \bar{y}^{\prime}\right) .
\end{aligned}
$$

Within this axiomatic framework, we are able to introduce the axiomatization of modal kinematics of special relativity:

MSpecRel $\stackrel{\text { def. }}{=}$

$\{$ AxEField, AxMFrame, AxPhExp, AxPhObs, AxMEv, AxSelf, AxMSym $\}$ 
Within this axiom system, we can prove all the kinematical effects of special relativity, such as time dilation and length contraction. See [3] for a direct proof for these effects in a classical axiomatic framework. Here, instead of proving these directly, we prove that worldview transformations are Poincaré transformations, which imply all kinematical effects of special relativity.

\section{Theorem 3.}

$$
\text { MSpecRel } \vdash(\forall k, h \in \mathrm{IOb}) \text { "w } k h \text { is a Poincaré transformation." }
$$

The proof is in Appendix 5.1.

\section{Dynamics}

From now on, we will assume MSpecRel without further mentioning.

\subsection{Definition of Mass}

In this section, we introduce the special relativistic dynamics based on kinematical notions. We base our definition of mass on possible collisions with the mass-standard. However, we have to give a definition for inertial bodies and collisions first. Instead of giving a definition generally for all type of collisions, we restrict ourselves to inelastic collisions involving only two bodies. This does not mean that our dynamics is applicable only to these types of collisions. The method can easily be generalized, see [31]. The reason why we choose these simple collisions is that they give a sufficient basis to define the relativistic mass explicitly.

Definition 3 (Inertial bodies and their speed). A body is inertial iff its worldline can be covered by a line:

$$
\begin{aligned}
\operatorname{IB}(b) \stackrel{\text { def. }}{\Longleftrightarrow}(\exists k \in \mathrm{IOb})\left(\forall \bar{x}, \bar{y}, \bar{z} \in \mathrm{wline}_{k}(b)\right) \\
\\
\quad\left(\bar{x}_{t} \leq \bar{y}_{t} \leq \bar{z}_{t} \rightarrow|\bar{x}-\bar{y}|+|\bar{y}-\bar{z}|=|\bar{x}-\bar{z}|\right) .
\end{aligned}
$$

If a body $b$ is inertial and exists in at least two coordinate points, the following definition of speed is well-defined:

$$
\mathrm{v}_{k}(b)=v \stackrel{\text { def. }}{\Longleftrightarrow}\left(\exists x, y \in \text { wline }_{k}(b)\right)\left(x \neq y \wedge v=\frac{\operatorname{Space}(x, y)}{\operatorname{Time}(x, y)}\right) .
$$

Two examples for inertial bodies are the inertial observers (by AxSelf), and the photons (by AxPhObs), so IOb $\subseteq$ IB and $\mathrm{Ph} \subseteq$ IB. However, our intention with the definition of inertial bodies is to introduce the type of bodies to which we would like to assign mass. So first, inertial observers (i.e., coordinatesystems) are not such entities. Second, for simplicity, in this paper we will not consider the mass of photons. Therefore, we introduce the following notion for other inertial bodies.

Definition 4 (Ordinary body). We call a body ordinary iff it is an inertial body which is not a photon nor an inertial observer:

$$
\mathrm{OIB}(b) \stackrel{\text { def. }}{\Longleftrightarrow} \operatorname{IB}(b) \wedge \neg \mathrm{IOb}(b) \wedge \neg \mathrm{Ph}(b) .
$$


For ordinary bodies other than the mass-standard ${ }^{16}$, we introduce the following notation:

$$
\mathrm{OIB}^{-}(b) \stackrel{\text { def. }}{\Longleftrightarrow} \mathrm{OIB}(b) \wedge b \neq \varepsilon .
$$

Definition 5 (Collision). See Fig. 5. We say that $b$ and $c$ collide inelastically resulting a body $d$ according to an observer $k$ at the spacetime location $\bar{x}$, in formula inecoll $k, \bar{x}(b, c: d)$, iff $b$ and $c$ are different existing inertial bodies and their worldlines end in $\bar{x}$, and the worldline of the existing inertial body $d$ begins also in $\bar{x}$ according to $k$.

$$
\begin{aligned}
\operatorname{in}_{k}(\bar{x}) \stackrel{\text { def. }}{=}\left\{b \in \mathrm{IB}: b \in \mathrm{ev}_{k}(\bar{x}) \wedge\left(\forall \bar{y} \in \text { wline }_{k}(b)\right)\left(\bar{y}_{t}<\bar{x}_{t} \vee \bar{y}=\bar{x}\right)\right\}, \\
\operatorname{out}_{k}(\bar{x}) \stackrel{\text { def. }}{=} \quad\left\{b \in \mathrm{IB}: b \in \mathrm{ev}_{k}(\bar{x}) \wedge\left(\forall \bar{y} \in \text { wline }_{k}(b)\right)\left(\bar{y}_{t}>\bar{x}_{t} \vee \bar{y}=\bar{x}\right)\right\},
\end{aligned}
$$

inecoll $_{k, \bar{x}}(b, c: d) \stackrel{\text { def. }}{\Longleftrightarrow} b, c, d \in \mathrm{E} \wedge b \neq c \wedge \operatorname{in}_{k}(\bar{x})=\{b, c\} \wedge \operatorname{out}_{k}(\bar{x})=\{d\} .{ }^{17}$

The omitted variables are intended to be quantified over existentially:

$$
\begin{array}{rll}
\text { inecoll }_{k, \bar{x}}(b, c) & \stackrel{\text { def. }}{\Longleftrightarrow} & (\exists d \in \mathrm{IB}) \operatorname{inecoll}_{k, \bar{x}}(b, c: d), \\
\text { inecoll }_{k}(b, c) & \stackrel{\text { def. }}{\Longleftrightarrow} & (\exists \bar{x}) \text { inecoll }_{k, \bar{x}}(b, c), \\
\text { inecoll }(b, c) & \stackrel{\text { def. }}{\Longleftrightarrow} & \left(\exists k \in \mathrm{IOb}_{\text {inecoll }}(b, c) .\right.
\end{array}
$$

Figure 5: $\operatorname{in}_{k}(\bar{x}), \operatorname{out}_{k}(\bar{x})$ and inelastic collision
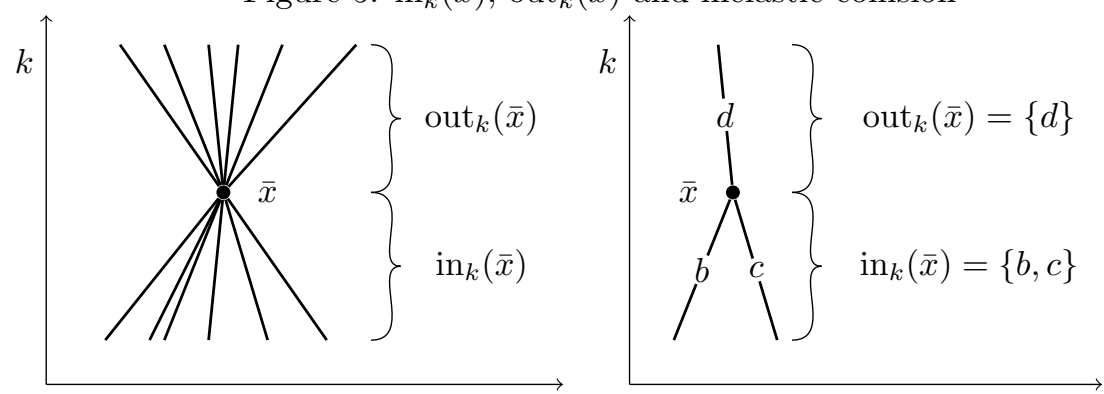

We also introduce a notation for the spacetime location of collisions:

$$
\operatorname{locinecoll}_{k}(b, c)=\bar{x} \stackrel{\text { def. }}{\Longleftrightarrow} \operatorname{inecoll~}_{k, \bar{x}}(b, c) .
$$

Let us note that, by the definition of inecoll, $\operatorname{locinecoll}_{k}(b, c)$ is well-defined.

Definition 6 (Covering line of inertial bodies). The covering line of inertial body $d$ according to observer $k$ is the line $\overline{\text { wline }}_{k}(d)$ which contains the worldline of $d$.

$$
\bar{z} \in \overline{\operatorname{wline}}_{k}(d) \stackrel{\text { def. }}{\Longleftrightarrow}\left(\forall \bar{x}, \bar{y} \in \operatorname{wline}_{k}(d)\right)\left(\begin{array}{l}
|\bar{x}-\bar{y}|+|\bar{y}-\bar{z}|=|\bar{x}-\bar{z}| \vee \\
|\bar{x}-\bar{z}|+|\bar{z}-\bar{y}|=|\bar{x}-\bar{y}| \vee \\
|\bar{z}-\bar{x}|+|\bar{x}-\bar{y}|=|\bar{z}-\bar{y}|
\end{array}\right)
$$

\footnotetext{
${ }^{16}$ We do not assume that the mass-standard is inertial in general. However, in thought experiments which we use to derive our theorems, the mass-standard will always be inertial. This will follow from axiom AxDir, see Axiom 9.

${ }^{17}$ These relations can also be defined in the object language using the method of footnote 13 together with $\operatorname{in}_{k}(\bar{x})=\{b, c\} \Longleftrightarrow a \in \operatorname{in}_{k}(\bar{x}) \leftrightarrow(a=b \vee a=c)$ and with a similar substitution for out $_{k}(\bar{x})$.
} 
Figure 6: The Collision Ratio

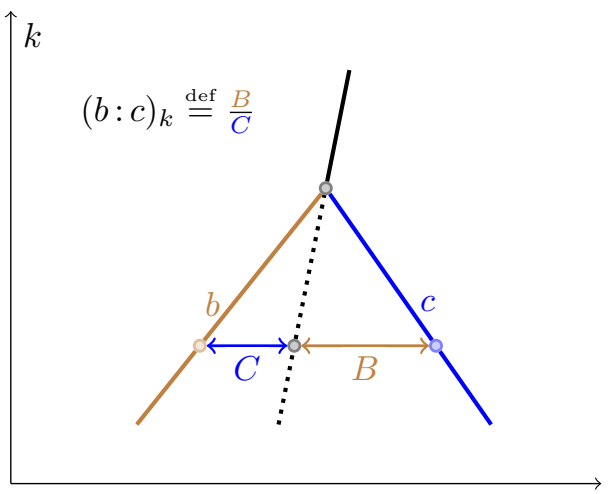

For inertial bodies participating in inelastic collisions, we can use the following notation since the covering line of these bodies cannot be horizontal:

$$
\begin{aligned}
& \text { wline }_{k}(d, t)=\bar{s} \stackrel{\text { def. }}{\Longleftrightarrow}\langle t, \bar{s}\rangle \in \text { wline }_{k}(d), \\
& \overline{\text { wline }}_{k}(d, t)=\bar{s} \stackrel{\text { def. }}{\Longleftrightarrow}\langle t, \bar{s}\rangle \in \overline{\text { wline }}_{k}(d) .
\end{aligned}
$$

How could we decide which one of two colliding bodies, say $b$ and $c$, is more massive? We can observe the resulting body $d$ of the collision: if $d$ is stationary, then the relativistic masses of $b$ and $c$ are equal; if $d$ moves towards where from $c$ have arrived, then $b$ is more massive than $c$. So we can examine the ratio of the covering lines of the bodies $b, c$ and $d$ intersected with the simultaneity of an observer $k$, see Fig. 6 . If this ratio is greater than 1 , then $b$ is more massive; and if this ratio is say 2.7, then $b$ is 2.7 times more massive than $c$.

We will define the ratio of collision only for those collisions in which the resulting body's worldline is between the two colliding ordinary bodies, like in Fig. 6. Formally:

$$
\begin{aligned}
& \operatorname{Between}_{k}(b, d, c) \stackrel{\text { def. }}{\Longleftrightarrow} \\
& \left(\forall \bar{x} \in \overline{\operatorname{wline}}_{k}(b)\right)\left(\forall \bar{y} \in \overline{\text { wline }}_{k}(c)\right)\left(\forall \bar{z} \in \overline{\text { wline }}_{k}(d)\right)(\exists t) \\
& {[0<t<1 \wedge \bar{z}=t \bar{x}+(1-t) \bar{y}] \vee \bar{x}=\bar{y}=\bar{z} .}
\end{aligned}
$$

Definition 7 (Ratio of Collision). We say that $b$ is $r$ times more massive than $c$ according to $k$, and we denote this by $(b: c)_{k}=r$, iff the covering line of the resulting body of the collision produced by $b$ and $c$ divides the simultaneity of $k$ between the body $b$ and $c$ in the ratio of $r$ :

$$
\begin{aligned}
(b: c)_{k}=r \stackrel{\text { def. }}{\Longleftrightarrow} & \operatorname{inecoll}(b, c) \wedge \operatorname{Between}_{k}(b, d, c) \wedge \\
& \wedge\left(\exists t<\operatorname{locinecoll}_{k}(b, c)\right) \quad r=\frac{\left|\overline{\operatorname{wline}}_{k}(c, t)-\overline{\mathrm{wline}}_{k}(d, t)\right|}{\left|\overline{\mathrm{wline}}_{k}(b, t)-\overline{\mathrm{wline}}_{k}(d, t)\right|} .
\end{aligned}
$$

Having the notion of ratio of collision we are close to define a relativistic mass function: If $c$ is the mass-standard $\varepsilon$, then $(b: \varepsilon)_{k}=r$ should be read as 
" $b$ is $r$ times more massive than the mass-standard according to $k$." And what else would we like to understand by that " $b$ has the relativistic mass $r$ according to $k$ " if not this? ${ }^{18}$

However, such a definition for mass seems to be too narrow. There are three problems with this definition:

(1) Problem of the Non-interacting. How could we say anything about bodies that do not collide with something? Do they lack mass? Even if we do not know about the mass of such a body, it should have mass or at least it should be meaningful to speak about its mass.

(2) Problem of Reusability. If the mass-standard collides with a body, how could it collide again if we use only inelastic collisions?

(3) Problem of the Stationary. The mass-standard should have the mass 1 only if it is stationary since relativistic mass, similarly to length and time, depends on speed in relativity theory. What if $b$ is at rest, too? How could such a stationary $b$ be collided with the mass-standard if the mass-standard is also stationary?

We solve these problems using possible world semantics and thought experiments. (1) can be solved by speaking about collisions in alternative possible worlds where it collides with the mass-standard instead of the actual world where it does not. This also solves (2): the actual world can be counted as the first use of the mass-standard, and the alternative world can be the second use. And similarly, every other measurement (collision with the mass-standard) can be done in another alternative world of the actual one.

So shortly: to define relativistic mass we will use collisions in alternative possible worlds. We can summarize the answer to the first two problems in a sketch of a definition of mass for moving bodies:

"Definition" 1 (Mass of the Moving). The relativistic mass of a moving ordinary body $b$ according to an observer $k$ is $r$, iff it could be $r$ times more massive than the mass-standard: there is a "very similar" alternative world in which $b$ collides with the mass-standard with the collision ratio of $(b: \varepsilon)_{k}=r$.

We can also solve (3) using this quasi definition, i.e., we can define rest mass based on the mass of moving bodies by using a transmitting body between the stationary mass-standard and the stationary body which is going to be measured:

"Definition" 2 (Mass of the Stationary). The relativistic mass of a stationary body $b$ is $r_{1} \cdot r_{2}$ according to $k$ iff it could be $r_{1}$ times more massive than a body which could be $r_{2}$ times more massive than the mass-standard: There is a "very

\footnotetext{
${ }^{18}$ Practically, the ratio of collision is a formal implementation of Weyl's definition for ratios of masses, see $[21,(1.4)$ on p.10.], implemented to special relativity. Suppose that we already have a mass function $m$ having the usual properties. So $m_{k}(b)$ denotes the relativistic mass of $b$ according to $k$. Let $b$ and $c$ be two colliding bodies, and $k$ be the inertial observer according to which the center of mass of $b$ and $c$ is stationary. Then $(b: c)_{k}$ is the ratio $\mathrm{v}_{k}(c) / \mathrm{v}_{k}(b)$. Therefore, the collision ratio $(b: c)_{k}$ corresponds to the ratio $m_{k}(b) / m_{k}(c)$ by the conservation of linear momentum. And since Poincaré transformations preserve the ratio of points on a line, the ratio of collision means the ratio of masses even if we choose a different observer than $k$.
} 
similar" alternative possible world in which $b$ collides with a (moving) body $c$ with the collision ratio $(b: c)_{k}=r_{1}$ and the relativistic mass of $c$ is $r_{2}$.

There is only one problem with these two quasi definitions: What does it mean that the alternative world is "very similar" to the actual one? Not any kind of world is relevant if we want to collide the mass-standard to a body $b$. We are interested only in those worlds where $b$ has the same speed. These considerations motivate the following two semantical definitions.

Since in modal logic, the predicates can vary in different worlds, when it is not straightforwardly determined by the context, we label the predicates by worlds. So here and from now on, superscript $w$ in predicates $P^{w}$ and terms $t^{w}$ denotes the worlds from which we took it.

Definition 8 (Collision Thought Experiments and their Relevance). We say that in world $w$ body $c$ can collide with $b$ according to $k$ iff $b$ is an existing ordinary body and $k$ is an existing observer in $w$, and there is an alternative world $w^{\prime}$ where these are still existing, inertial, the observer is still an observer, and there $c$ is an existing ordinary body colliding with $b$. Formally, in $w$ a body $c$ can collide with $b$ according to $k$ iff

$$
\begin{aligned}
& \left(\exists w^{\prime} \in S\right) w R w^{\prime}, \\
& b \in D_{w} \cap \mathrm{OIB}^{\mathfrak{M}, w} \cap D_{w^{\prime}} \cap \mathrm{OIB}^{\mathfrak{M}, w^{\prime}}, \\
& c \in \mathrm{OIB}^{\mathfrak{M}, w^{\prime}} \cap D_{w^{\prime}}, \\
& k \in \mathrm{IOb}^{\mathfrak{M}, w} \cap D_{w} \cap \operatorname{IOb}^{\mathfrak{M}, w^{\prime}} \cap D_{w^{\prime}}, \\
& \left(\exists \bar{x} \in \operatorname{wline}_{k}(b)_{w}\right) \operatorname{inecoll}_{k, \bar{x}}(b, c)_{w^{\prime}} .
\end{aligned}
$$

We call such a $\left\langle w, w^{\prime}, k, b, c\right\rangle$ tuple a collision thought experiment or just collision experiment.

We call a collision thought experiment $\left\langle w, w^{\prime}, k, b, c\right\rangle$ relevant iff the worldline of $b$ before the collision is the same in both worlds according to $k$.

$$
\left(\forall t \leq \operatorname{locinecoll}_{k}(b: c)_{t}^{w}\right) \quad \text { wline }_{k}(b, t)^{w}=\text { wline }_{k}(b, t)^{w^{\prime}} .
$$

The following axiom ensures that all collision thought experiments are relevant:

Axiom 8 (Axiom of Relevant Collisions).

AxCollRel Every collision thought experiment is relevant:

$$
\begin{aligned}
& (\forall k \in \mathrm{IOb})(\forall b \in \mathrm{OIB})(\forall \bar{x}, \bar{y}) \\
& \quad\left(\bar{y}_{t} \leq \bar{x}_{t} \wedge \mathrm{W}(k, b, \bar{y})\right) \rightarrow \square\left((\exists c \in \mathrm{OIB}) \text { inecoll }_{k, \bar{x}}(b, c) \rightarrow \mathrm{W}(k, b, \bar{y})\right) .
\end{aligned}
$$

This axiom is the engine of our Dynamics. Upon colliding an ordinary body $c$ to an ordinary body $b$, we assume: The worldline of $b$ changes after the collision and remains unchanged before the collision (otherwise its speed could change and that would ruin the whole experiment). So this axiom erases the worldline after a certain point to make room for the collision, but preserve the rest of the worldline to maintain the speed. This axiom also ensures a very important fact: the relative speed of two observers remains the same in collision thought experiments, see Item 1. of Prop. $4 .{ }^{19}$

Now that we are able to filter out the relevant collisions, we can introduce collision experiments designed to determine the masses of the moving and stationary bodies.

\footnotetext{
${ }^{19}$ The reader may wonder why is the formula of AxCollRel so complicated, while the informal
} 
Figure 7: Direct Measurement

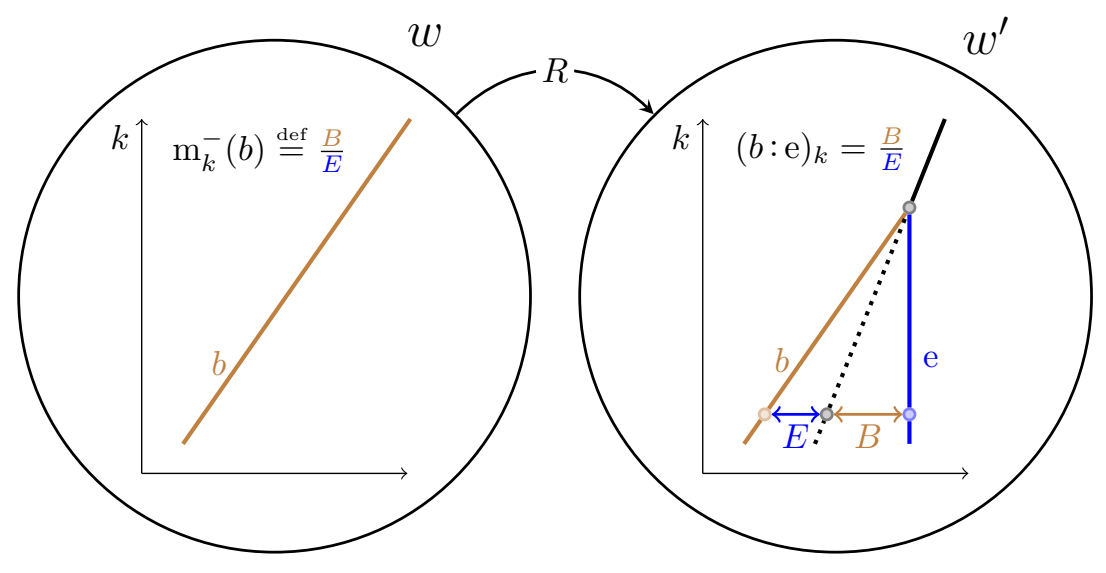

Figure 8: Indirect Measurement

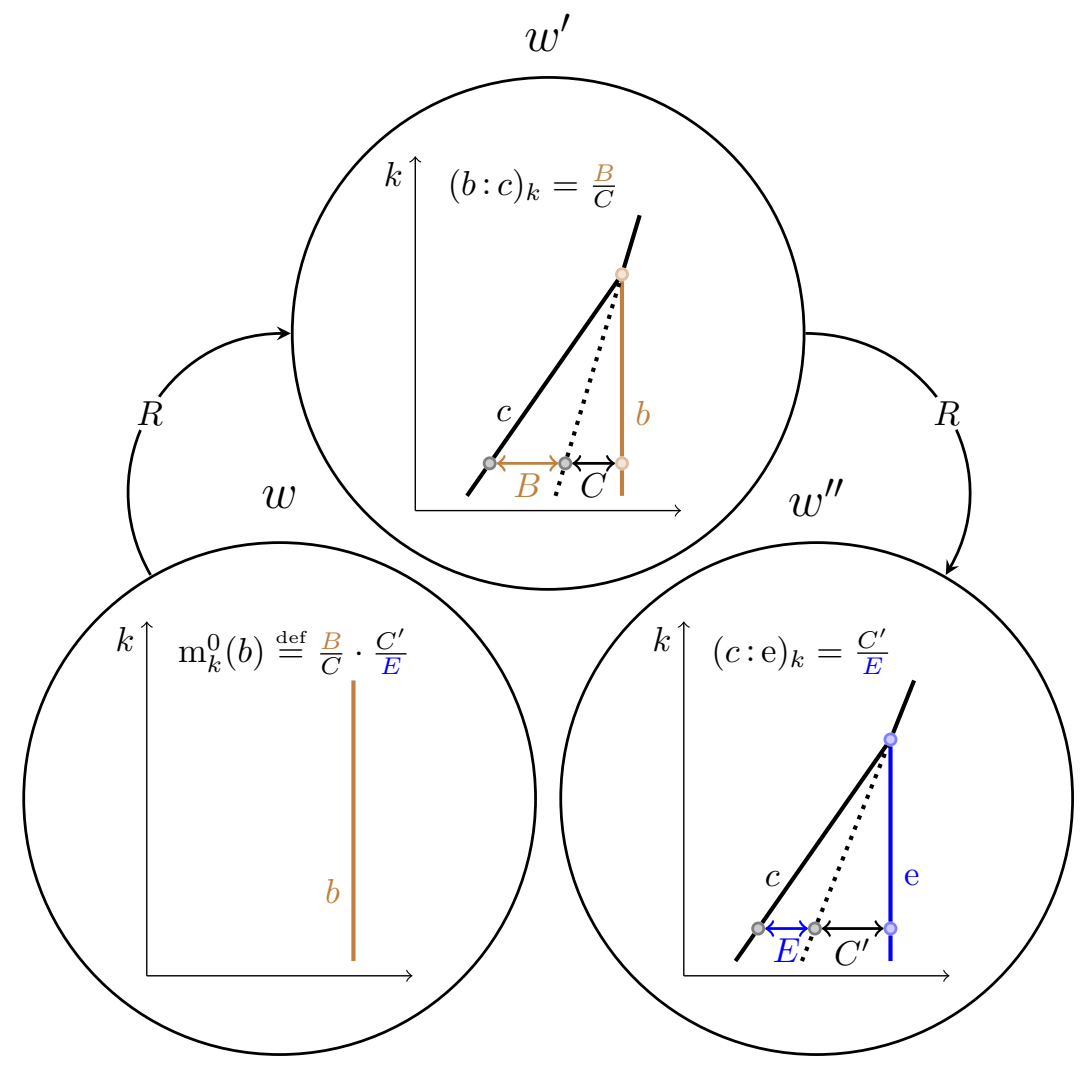


Definition 9 (Measurements). A collision experiment $\left\langle w, w^{\prime}, k, b, c\right\rangle$ is a direct measurement iff it is relevant, $c$ is the mass-standard and it is stationary according to $k$. See Fig. 7. A collision experiment $\left\langle w, w^{\prime}, k, b, c\right\rangle$ is an indirect measurement iff it is relevant, $b$ is stationary, and there exists a direct measurement $\left\langle w^{\prime}, w^{\prime \prime}, k, c, \varepsilon\right\rangle$. See Fig. 8 .

The following two axioms will ensure that the measurements described above are determined uniquely.

Axiom 9 (Axiom of Direct Measurements).

AxDir According to any observer, every relatively moving ordinary body, other than the mass-standard, can uniquely collide with the mass-standard such that the mass-standard is stationary for that observer:

$(\forall k \in \mathrm{IOb})\left(\forall b \in \mathrm{OIB}^{-}\right)$

$$
\mathrm{v}_{k}(b) \neq 0 \rightarrow(\exists r)\left(\begin{array}{r}
\diamond\left[\mathrm{v}_{k}(\varepsilon)=0 \wedge(b: \varepsilon)_{k}=r\right] \wedge \\
\wedge \square\left[\mathrm{v}_{k}(\varepsilon)=0 \rightarrow(b: \varepsilon)_{k}=r\right]
\end{array}\right) .
$$

If AxDir is assumed, we can define the mass of relatively moving ordinary bodies (except the mass-standard) as it was illustrated on Fig. $7:{ }^{20}$

$$
\mathrm{m}_{k}^{-}(b)=r \stackrel{\text { def. }}{\Longleftrightarrow} \diamond\left[\mathrm{v}_{k}(\varepsilon)=0 \wedge(b: \varepsilon)_{k}=r\right] .
$$

Axiom 10 (Axiom of Indirect Measurements).

AxIndir For every observer, every stationary ordinary body is involved in an indirect measurement, and the results of indirect measurements are unique, i.e., do not depend on the choices of the transmitting body: ${ }^{21}$

$$
\begin{aligned}
& (\forall k \in \mathrm{IOb})(\forall b \in \mathrm{OIB}) \\
& \qquad \begin{aligned}
\mathrm{v}_{k}(b) & =0 \rightarrow(\exists r)\left[\diamond ( \exists c \in \mathrm { OIB } ^ { - } ) \left(r=(b: c)_{k} \cdot \mathrm{m}_{k}^{-}(c) \wedge\right.\right. \\
& \left.\left.\wedge \square\left(\forall c^{\prime} \in \mathrm{OIB}^{-}\right)\left(\operatorname{inecoll}\left(b, c^{\prime}\right) \rightarrow r=\left(b: c^{\prime}\right)_{k} \cdot \mathrm{m}_{k}^{-}\left(c^{\prime}\right)\right)\right)\right] .
\end{aligned}
\end{aligned}
$$

If AxDir and AxIndir are assumed, we can define the mass of stationary ordinary bodies as it was illustrated on Fig. 8:

$$
\mathrm{m}_{k}^{0}(b)=r \stackrel{\text { def. }}{\Longleftrightarrow} \mathrm{v}_{k}(b)=0 \wedge \diamond(\exists c \in \mathrm{OIB})\left[r=(b: c)_{k} \cdot \mathrm{m}_{k}^{-}(c)\right] .
$$

description is so simple, what is more, why do we postulate that every collision thought experiment is relevant instead of only those that we really need? The reason is that the expressive power of first-order modal logic is not as strong as it seems. For example, it is hopeless to show a formula expressing exactly the following: "There is an alternative world $w^{\prime}$ in which every object from $w$ having property $P_{w}$, has a property $Q_{w^{\prime}}$ in $w^{\prime}$." The main reason for this is that we cannot 'quantify back' into the previous world after we used a $\diamond$ operator. For a summary of expressivity problems of first-order modal logic, see [17], [19]. Now the dynamical statement like "Only b's worldline changes" is also such a statement. So this control is beyond the expressibility power of first-order modal logic. At the conference LR12 [26] and in [27], we sketched a solution which used a trick to enforce this kind of thought experiments, but it cost a lot: it used two modal operators such that one of them was a transitive closure of the other. A strong completeness theorem for such a logic is impossible, see $[9, \S 4.8$ Finitary Methods I.]. So AxCollRel seems to be the appropriate axiom which makes relevant collision thought experiments possible, and is still expressible.

${ }^{20}$ Note that the definitions (3), (4) and (5) express their intended meanings only if we assume AxCollRel as well.

${ }^{21}$ It is a question for further research to find natural and more elementary axioms implying that the results of indirect measurements do not depend on the choices of transmitting body. 
We can define an observer-independent concept of rest mass as well:

$$
\mathrm{m}_{0}(b)=r \stackrel{\text { def. }}{\Longleftrightarrow}(\exists k \in \mathrm{IOb}) \quad \mathrm{m}_{k}^{0}(b)=r .
$$

To show that $\mathrm{m}_{0}(b)$ is a well-defined quantity, we have to prove that $\mathrm{m}_{k}^{0}(b)$ does not depend on $k$, i.e., co-moving observers get the same results from indirect measurements. We prove this in four steps:

\section{Proposition 4.}

1. The relative speed of observers remains the same in collision experiments, i.e., thought experiments described in Def. 8.

$$
\begin{aligned}
& \text { MSpecRel } \cup\{\text { AxCollRel }\} \\
& \qquad \\
&(\forall k, h \in \mathrm{IOb})(\forall r)(\forall b \in \mathrm{OIB})\left[\mathrm{v}_{k}(h)=r \rightarrow\right. \\
&\left.\rightarrow \square\left((\exists c \in \mathrm{OIB}) \operatorname{inecoll}(b, c) \rightarrow \mathrm{v}_{k}(h)=r\right)\right] .
\end{aligned}
$$

2. In collision experiments, ordinary bodies have the same collision ratio for every two inertial observers co-moving with each other.

$$
\begin{aligned}
& \text { MSpecRel } \cup\{\text { AxCollRel }\} \vdash \\
& (\forall k, h \in \mathrm{IOb})\left[\mathrm{v}_{k}(h)=0 \rightarrow(\forall b \in \mathrm{OIB}) \square(\forall c \in \mathrm{OIB})(b: c)_{k}=(b: c)_{h}\right] .
\end{aligned}
$$

3. Inertial observers co-moving with each other get the same results in direct measurements.

$$
\begin{aligned}
& \text { MSpecRel } \cup\{\text { AxCollRel, AxDir }\} \vdash \\
& (\forall k, h \in \mathrm{IOb})\left[\mathrm{v}_{k}(h)=0 \rightarrow\left(\forall b \in \mathrm{OIB}^{-}\right)\left(\mathrm{v}_{k}(b) \neq 0 \rightarrow \mathrm{m}_{k}^{-}(b)=\mathrm{m}_{h}^{-}(b)\right)\right] .
\end{aligned}
$$

4. Inertial observers co-moving with each other get the same results in indirect measurements.

$$
\begin{aligned}
& \text { MSpecRel } \cup\{\text { AxCollRel, AxDir, AxIndir }\} \vdash \\
& \qquad(\forall k, h \in \mathrm{IOb})(\forall b \in \mathrm{OIB}) \mathrm{m}_{k}^{0}(b)=\mathrm{m}_{h}^{0}(b) .
\end{aligned}
$$

Proof.

1.: Let $w$ be an arbitrary but fixed world in which $k$ and $h$ are inertial observers moving with the speed of $\mathrm{v}_{k}(h)=\mathrm{v}_{h}(k)=r$. Let $b$ an ordinary body from $w$. Let $w^{\prime}$ an arbitrary but fixed transformed version of $w$ in which $b$ collides inelastically with an ordinary body $c$. From AxMFrame, we know that both $k$ and $h$ exist as observers in $w^{\prime}$, too. From now on, we omit the details concerning AxMFrame in this proof.

We have to prove that $\mathrm{v}_{k}(h)=r$ in $w^{\prime}$, too. To prove that, by Thm. 3 , it is enough to show that the transformations $\mathrm{w}_{k h}{ }^{w}$ and $\mathrm{w}_{k h}{ }^{w^{\prime}}$, the worldview transformation in $w$ and the worldview transformation in $w^{\prime}$, take one timelike line to the same line, i.e.,

$$
\mathrm{w}_{k h}{ }^{w^{\prime}}[\ell]=\mathrm{w}_{k h}{ }^{w}[\ell] .
$$


For such a line, the covering line of $b$ for $k$ is a perfect choice, since $\left\langle w, w^{\prime}, k, b, c\right\rangle$ is a collision thought experiment, and by AxCollRel, it is relevant:

$$
\begin{aligned}
& \mathrm{w}_{k h}^{w^{\prime}}\left[\overline{\mathrm{wline}}_{k}(b)^{w^{\prime}}\right]= \overline{\mathrm{wline}}_{h}(b)^{w^{\prime}} \stackrel{\text { AxCollRel }}{\underline{\downarrow}} \overline{\mathrm{wline}}_{h}(b)^{w}= \\
& \mathrm{w}_{k h}^{w}\left[\overline{\mathrm{wline}}_{k}(b)^{w}\right] \stackrel{\text { AxCollRel }}{\stackrel{\downarrow}{=}} \quad \mathrm{w}_{k h}^{w}\left[\overline{\mathrm{wline}}_{k}(b)^{w^{\prime}}\right] .
\end{aligned}
$$

2.: Let $w$ be an arbitrary but fixed world in which $k$ and $h$ are existing comoving observers coordinatizing an ordinary body $b$. Let $w^{\prime}$ an arbitrary but fixed transformed version of $w$, in which $b$ collides inelastically with an ordinary body $c$.

Let $d$ be the resulting body of the collision. To prove $(b: c)_{k}=(b: c)_{h}$, it is enough to show in $w^{\prime}$, that the covering lines of $b, c$ and $d$ according to $k$ are parallel to the covering lines of $b, c$ and $d$ according to $h$, respectively. By Thm. 3 and AxSelf, assumption $\mathrm{v}_{k}(h)=0$ ensures this in $w$. So we need that $\mathrm{v}_{k}(h)=0$ in $w^{\prime}$ as well. But this follows from Item 1.

3.: Let $w$ be a world in which $k$ and $h$ are co-moving existing inertial observers cooordinatizing an ordinary body $b$ moving.

By AxDir and AxCollRel, " $k$ can measure" the mass of $b$ directly, i.e., there is a world $w^{\prime}$ such that $w R w^{\prime}$ and in $w^{\prime}$ the stationary mass-standard $\varepsilon$ collides with $b$ with a unique collision ratio $r=(b: \varepsilon)_{k}$. Because $r$ is unique by AxDir, by using Item 2, we have:

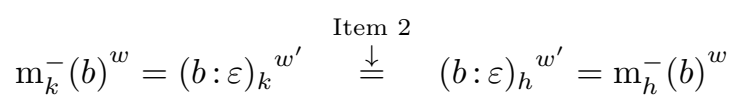

4.: Let $w$ be a world in which $k$ and $h$ are co-moving observers cooordinatizing an ordinary body $b$ stationary.

By AxIndir and AxCollRel, $k$ can measure the mass of $b$ indirectly, i.e., there is a world $w^{\prime}$ such that $w R w^{\prime}$ and in $w^{\prime}$, a transmitting ordinary body $c$ collides with $b$ with a collision ratio $r=(b: c)_{k}$. By Item $2, r=(b: c)_{h}$ in $w^{\prime}$ as well. By Item $3, \mathrm{~m}_{k}^{-}(c)=\mathrm{m}_{h}^{-}(c)$ in $w^{\prime}$. Since the result of the indirect measurement is unique because of AxIndir, we have the equations

$$
\mathrm{m}_{k}^{0}(b)_{w}=(b: c)_{k} \cdot \mathrm{m}_{k}^{-}(c)^{w^{\prime}} \stackrel{\text { Item } 2,3}{\stackrel{\downarrow}{=}}(b: c)_{h} \cdot \mathrm{m}_{h}^{-}(c)^{w^{\prime}}=\mathrm{m}_{h}^{0}(b)^{w}
$$

Definition 10 (Relativistic Mass). Assume AxDir and AxIndir. We define the relativistic mass of ordinary body $b$ according to observer $k$ by putting definitions (3) and (4) together:

$$
\mathrm{m}_{k}(b) \stackrel{\text { def. }}{=}\left\{\begin{array}{l}
\mathrm{m}_{k}^{-}(b), \text { if } \mathrm{v}_{k}(b) \neq 0 \\
\mathrm{~m}_{k}^{0}(b) \text { otherwise. }
\end{array}\right.
$$


Because we defined the mass with the ratios of collision, we have a restricted "built-in" conservation-theorem: a restricted conservation of the centerline of mass.

Proposition 5. Assume AxDir and AxIndir. If $c$ is at rest according to $k$, and $b$ and $c$ are colliding ordinary bodies, such that $b$ is different from the massstandard, then the collision ratio of this collision is the ratio of their masses.

$$
\begin{aligned}
& \text { MSpecRel } \cup\{\text { AxDir, AxIndir }\} \vdash \\
& \qquad(\forall k \in \mathrm{IOb})\left(\forall b \in \mathrm{OIB}^{-}\right)(\forall c \in \mathrm{OIB})\left[\mathrm{v}_{k}(c)=0 \rightarrow\left((b: c)_{k}=\frac{\mathrm{m}_{k}(b)}{\mathrm{m}_{k}(c)}\right)\right]
\end{aligned}
$$

Proof. By the definition of collision ratio, for all inertial observers $k$ and inertial bodies $b$ and $c$ :

$$
(b: c)_{k}=\frac{1}{(c: b)_{k}} .
$$

By equation (6), we have the following chain of equations:

$$
(b: c)_{k}=\frac{1}{(c: b)_{k}}=\frac{\mathrm{m}_{k}^{-}(b)}{(c: b)_{k} \cdot \mathrm{m}_{k}^{-}(b)}=\frac{\mathrm{m}_{k}^{-}(b)}{\mathrm{m}_{k}^{0}(c)}=\frac{\mathrm{m}_{k}(b)}{\mathrm{m}_{k}(c)} .
$$

So the collision ratio is determined by the masses of $b$ and $c$, or in other words, the worldline of the resulting body is the continuation of the center of masses of the colliding bodies. So the centerline is conserved in the following sense: the covering line of the center of masses is the same as the covering line of the resulting body.

However, this conservation theorem is restricted: we proved only with the premise that one of the colliding bodies is at rest according to the observer. The more general statement, called AxCenter, in which the colliding bodies can both be moving according to the observer is the key axiom in [4], [23], [31, §5]. It is an interesting fact that in the modal framework we can prove the Mass Increase Theorem even without this more general assumption. ${ }^{22}$

\subsection{Equivalents of the mass-standard}

We need one more tool for measurement: the experiments with mass-standardequivalents. These are, as their name says, introduced with the purpose to replace or substitute the mass-standard. We need such tools usually when we need more measuring tools than the only mass-standard, e.g., when two relatively moving observers try to compare their measuring results.

\footnotetext{
${ }^{22}$ Proving that this key axiom does not follow even from our axiom system MSpecRelDyn (see below on p.27) is out of the scope of this paper, since it involves an even more complicated model construction than what is outlined in Appendix 5.3. Nevertheless, the key idea is that if we have three worlds $w, w_{1}$ and $w_{2}$ such that $w R w_{1}$ and $w R w_{2}$, then our only axioms that are capable of harmonizing the collisions in $w_{1}$ and $w_{2}$ are AxDir, AxIndir and AxPDirComp (for the latter see the next section). However, if none of the colliding bodies are co-moving with an observer (to use AxIndir and AxPDirComp) or identical with the mass-standard (to use AxDir), then our axioms say nothing about the collisions in $w_{1}$ and $w_{2}$. This fact can be used to construct an appropriate model because AxCenter claims that if we have a collision in $w$, then the measurements in $w_{1}$ and $w_{2}$ are determined by $w$
} 
We have two main expectations about mass-standard-equivalents: they should be able to substitute the mass-standard $\varepsilon$ in an equivalent way, and they should have the rest masses 1 . Either property could be a good definition, but we start from a more basic level. We define mass-standard-equivalents by the following expectation: if a mass-standard-equivalent (whatever it is) collides with the mass-standard itself, then the resulting body should be stationary according to any median observer according to who these two bodies have opposite velocities.

Definition 11 (Median Observer). An inertial observer $m$ is median of the collision consisting body $b$ and $c$ iff the velocity of $b$ is the opposite of that of $c$ according to $m$ :

$$
\operatorname{MedianOb}_{b, c}(m) \stackrel{\text { def. }}{\Longleftrightarrow} \operatorname{inecoll}(b, c) \wedge \overline{\mathrm{v}}_{m}(b)+\overline{\mathrm{v}}_{m}(c)=\overline{0} .
$$

Definition 12 (Symmetric Collision). A collision is called symmetric iff there is a median observer of it coordinatizing the resulting body at rest:

$$
\operatorname{SymColl}(b, c) \stackrel{\text { def. }}{\Longleftrightarrow}\left(\exists m \in \operatorname{MedianOb}_{b, c}\right)(b: c)_{m}=1 .
$$

Definition 13 (Mass-standard-equivalents). A body $b$ is an equivalent of the mass-standard of $k$ iff $b$ is co-moving with $k$, different from the mass-standard, and whenever it collides with the mass-standard, it collides with it symmetrically.

$$
\mathrm{Et}_{k}(b) \stackrel{\text { def. }}{\Longleftrightarrow} \mathrm{OIB}^{-}(b) \wedge \mathrm{v}_{k}(b)=0 \wedge \square(\operatorname{inecoll}(b, \varepsilon) \rightarrow \operatorname{SymColl}(b, \varepsilon))
$$

Theorem 6 (Symmetric Collision Theorem). Symmetric collisions have the collision ratio of $\sqrt{1-v^{2}}$ according to the co-moving observer of one of the colliding bodies, where $v$ is the speed of the other body.

$$
\begin{aligned}
\text { MSpecRel } \vdash(\forall b, c \in \mathrm{OIB})(\forall k, h & \in \mathrm{IOb})([\operatorname{SymColl}(b, c) \wedge \\
\wedge \mathrm{v}_{k}(b) & \left.\left.=0 \wedge \mathrm{v}_{h}(c)=0\right] \rightarrow(b: c)_{k}=\sqrt{1-\mathrm{v}_{h}(k)^{2}}\right)
\end{aligned}
$$

For the proof of this theorem, see Appendix 5.2.

Proposition 7 (Relativistic mass of moving equivalents). Assume AxDir and AxCollRel. Every observer $k$ measures every relatively moving $h$ observer's equivalent(s) to be $\frac{1}{\sqrt{1-v_{k}\left(e_{h}\right)^{2}}}$.

$$
\begin{aligned}
& \text { MSpecRel } \cup\{\text { AxDir, AxCollRel }\} \vdash(\forall k, h \in \mathrm{IOb}) \\
& \qquad \mathrm{v}_{k}(h) \neq 0 \rightarrow\left(\forall \mathrm{e}_{h} \in \mathrm{Et}_{h}\right) \mathrm{m}_{k}\left(\mathrm{e}_{h}\right)=\frac{1}{\sqrt{1-\mathrm{v}_{k}(h)^{2}}}
\end{aligned}
$$

Proof. Let $w$ be a world in which there are two observers $k$ and $h$ and a massstandard-equivalent $e_{h}$ of $h$ such that $\mathrm{v}_{k}(h) \neq 0$. Since $e_{h}$ is stationary for $h$ by definition, it moves for $k$. Therefore, by AxDir, there is a possible world $w^{\prime}$ where $\mathrm{e}_{h}$ collides with the mass-standard $\varepsilon$ stationary for $k$. Since $e_{h}$ exists in both worlds and collides in $w^{\prime}$, Item 1 of Prop. 4 ensures that the speed $\mathrm{v}_{k}(h)$ is the same in $w$ and $w^{\prime}$. Since $\mathrm{e}_{h}$ is an equivalent of the mass-standard, i.e., 
it collides symmetrically with the mass-standard, we know from the theorem of symmetric collisions (Thm. 6) that in this world the collision ratio according to $k$ is

$$
\left(\varepsilon: \mathrm{e}_{h}\right)_{k}=\sqrt{1-\mathrm{v}_{k}(h)^{2}} \stackrel{\stackrel{(6)}{\iota}}{\Longleftrightarrow}\left(\mathrm{e}_{h}: \varepsilon\right)_{k}=\frac{1}{\sqrt{1-\mathrm{v}_{k}(h)^{2}}} .
$$

This is exactly the definition of the relativistic mass of $\mathrm{e}_{h}$ in $w$.

To prove the substitutivity of the mass-standard, and that the equivalents' rest masses are 1 we use the following assumption:

Axiom 11 (Axiom of Symmetry of Equivalents).

AxEqSym The equivalents of the mass-standard collide symmetrically with each other if there is a median observer of the collision:

$$
(\forall k, l \in \mathrm{IOb})\left(\forall \mathrm{e}_{k} \in \mathrm{Et}_{k}\right)\left(\forall \mathrm{e}_{l} \in \mathrm{Et}_{l}\right)
$$

$$
(\exists m) \operatorname{MedianOb}_{\mathrm{e}_{k}, \mathrm{e}_{l}}(m) \rightarrow \operatorname{SymColl}\left(\mathrm{e}_{k}, \mathrm{e}_{l}\right) .
$$

Furthermore, we postulate a thought experimentation axiom very similar to the axiom of direct measurements AxDir. The axiom of pseudo-direct measurement with comparison enables us to collide bodies with equivalents of the mass-standard (instead of the mass-standard, as AxDir does) in a way we can compare them with a median observer. This axiom comes handy, when we should collide "the mass-standard with itself."

Axiom 12 (Pseudo-Direct Experimentation with Comparison).

AxPDirComp According to any observer, every ordinary body can collide with one of the observer's mass-standard-equivalents. Moreover, if the body which is going to be collided is a mass-standard-equivalent as well, then a median observer stands ready to compare them:

$$
\begin{aligned}
(\forall k \in \mathrm{IOb})(\forall b \in \mathrm{OIB}) \diamond\left(\exists \mathrm{e}_{k} \in \mathrm{Et}_{k}\right)\left[\operatorname{inecoll}\left(b, \mathrm{e}_{k}\right) \wedge\right. \\
\left.\wedge\left((\exists l) \operatorname{Et}_{l}(b) \rightarrow(\exists m) \operatorname{MedianOb}_{b, \mathrm{e}_{k}}(m)\right)\right] .
\end{aligned}
$$

Now we can introduce axiom system MSpecRelDyn, which implies the Mass Increase Theorem.

MSpecRelDyn $\stackrel{\text { def }}{=}$

MSpecRel $\cup\{$ AxCollRel, AxDir, AxIndir, AxPDirComp, AxEqSym $\}$

MSpecRelDyn is consistent. For more details and some observations about the independence of the axioms, see Appendix 5.3.

Proposition 8 (Rest mass of mass-standard).

$$
\text { MSpecRel } \cup\{\text { AxCollRel, AxDir, AxIndir }\} \vdash \mathrm{m}_{0}(\varepsilon)=1
$$


Proof. Let $c$ be the body consisted in the indirect measurement measuring the rest mass of $\varepsilon$. Since the collided body is the mass-standard, and the alternative relation is reflexive, we do not have to go further than one world away:

$$
\mathrm{m}_{0}(\varepsilon)=(\varepsilon: c)_{k} \cdot(c: \varepsilon)_{k} \stackrel{\stackrel{(6)}{\perp}}{=} 1
$$

In special relativity, we are mostly interested in those situations, where there are at least two relatively moving observers. So from now on, we will refer to this assumption as $\exists 2 \mathrm{IOb}$ :

$$
\exists 2 \mathrm{IOb} \stackrel{\text { def. }}{\Longleftrightarrow}(\exists k, h \in \mathrm{IOb}) \mathrm{v}_{k}(h) \neq 0 .
$$

Proposition 9 (Rest mass of equivalents). The rest masses of equivalents of the mass-standard are 1 if there are two relatively moving inertial observers.

$$
\text { MSpecRelDyn } \vdash \exists 2 \mathrm{IOb} \rightarrow(\forall k \in \mathrm{IOb})\left(\forall \mathrm{e}_{k} \in \mathrm{Et}_{k}\right) \mathrm{m}_{0}\left(\mathrm{e}_{k}\right)=1
$$

Proof. By AxIndir and AxCollRel, the rest mass is well defined for every equivalent $\mathrm{e}_{k}$ of $k$. We determine the rest mass using a relatively moving observer's equivalent:

$$
\mathrm{m}_{0}\left(\mathrm{e}_{k}\right)=\mathrm{m}_{k}^{0}\left(\mathrm{e}_{k}\right)=\left(\mathrm{e}_{k}: \mathrm{e}_{h}\right)_{k} \cdot \mathrm{m}_{k}^{-}\left(\mathrm{e}_{h}\right) .
$$

By AxEqSym and therefore the theorem of symmetric collisions (Thm. 6) and its corollary (Prop. 7), this is

$$
\mathrm{m}_{0}\left(\mathrm{e}_{k}\right)=\sqrt{1-\mathrm{v}_{k}(h)^{2}} \cdot \frac{1}{\sqrt{1-\mathrm{v}_{k}(h)^{2}}}=1 .
$$

Now we prove that the equivalents of the mass-standard are also equivalent in a formal sense: the mass-standard can be substituted by its co-moving equivalents.

Proposition 10 (Mass-standard-equivalence). The mass-standard can be replaced by its co-moving equivalents if there are two relatively moving inertial observer.

$$
\begin{aligned}
\text { MSpecRelDyn } \vdash \exists 2 \mathrm{IOb} \rightarrow(\forall k \in \mathrm{IOb})\left(\forall \mathrm{e}_{k} \in \mathrm{Et}_{k}\right)\left(\forall b \in \mathrm{OIB}^{-}\right) \\
\overline{\mathrm{v}}_{k}\left(\mathrm{e}_{k}\right)=\overline{\mathrm{v}}_{k}(\varepsilon) \rightarrow\left(b: \mathrm{e}_{k}\right)_{k}=\mathrm{m}_{k}(b) .
\end{aligned}
$$

Proof. From Prop. 9, we know that the equivalents have exactly the same rest mass as the mass-standard. From Prop. 5, we also know that the mass of these bodies ( since $\mathrm{e}_{k}$ is at rest for $k$ ) determines the collision ratio. So the collision ratios $\left(b: \mathrm{e}_{k}\right)_{k}$ and $(b: \varepsilon)_{k}$ cannot be different if the mass-standard is at rest. The latter is the definition of $\mathrm{m}_{k}(b)$. 


\subsection{Mass Increase Theorem}

Theorem 11 (Mass Increase Theorem).

$$
\text { MSpecRelDyn } \vdash(\forall k \in \mathrm{IOb})\left(\forall b \in \mathrm{OIB}^{-}\right) \quad \mathrm{m}_{0}(b)=\sqrt{1-\mathrm{v}_{k}(b)^{2}} \cdot \mathrm{m}_{k}(b)
$$

Proof. If $b$ is at rest according to $k$, then the statement is true by Def. 10 . So let us assume that $b$ is moving according to $k$.

By AxIndir, AxDir and the definition of mass, there is an observer $h$ according to whom $b$ is at rest, and there is a "transmitting" body $c$ such that there is an alternative possible world $w^{\prime}$ such that $w R w^{\prime}$ and in $w^{\prime}$ the following equation is true by (4):

$$
\mathrm{m}_{0}(b)=\mathrm{m}_{h}^{0}(b)=(b: c)_{h} \cdot \mathrm{m}_{h}(c) .
$$

Also by AxIndir, $\mathrm{m}_{0}(b)$ is independent from the choice of the 'transmitting' body $c$. Since $k$ is moving in $h$ 's coordinate system, by AxPDirComp, we can use a mass-standard-equivalent of $k$ for such a transmitting body $c$. Thus

$$
\mathrm{m}_{0}(b)=\left(b: \mathrm{e}_{k}\right)_{h} \cdot \mathrm{m}_{h}\left(\mathrm{e}_{k}\right) \stackrel{\text { Prop. } 7}{\stackrel{\downarrow}{=}}\left(b: \mathrm{e}_{k}\right)_{h} \cdot \frac{1}{\sqrt{1-\mathrm{v}_{k}(h)^{2}}},
$$

since $v_{k}(h)=v_{h}(k)$. By $\mathrm{v}_{h}(b)=0$, we have $\mathrm{v}_{k}(b)=\mathrm{v}_{k}(h)$ and

$$
\mathrm{m}_{0}(b)=\left(b: \mathrm{e}_{k}\right)_{h} \cdot \frac{1}{\sqrt{1-\mathrm{v}_{k}(b)^{2}}} .
$$

To obtain the theorem from (7), we have to show that

$$
\left(b: \mathrm{e}_{k}\right)_{h}=\left(b: \mathrm{e}_{k}\right)_{k} \cdot\left(1-\mathrm{v}_{k}(b)^{2}\right) .
$$

Let us now consider $k$ 's coordinate system on Fig. 9. Let $O$ be the coordinate point of the collision, and let $A$ be a coordinate point on the very same spatial location but 'one second earlier,' i.e., $A_{s}=O_{s}$ and $A_{t}=O_{t}-1$ (so $A$ is on $\varepsilon_{k}$ 's covering line). Let $B$ the coordinate point on $b$ 's covering line which is simultaneous to $A$ according to $k$, i.e., $B_{t}=A_{t}$. Then $|B A|=\operatorname{Space}(B, A)=$ $\mathrm{v}_{h}(b)$ since $\operatorname{Time}(O, A)=1$.

Let $E_{k}$ be the coordinate point from the covering line of $e_{k}$ which is 'simultaneous' for $h$, i.e., $\mathrm{w}_{k h}\left(E_{k}\right)_{t}=\mathrm{w}_{k h}(B)_{t}$. Then triangles $A O B$ and $A E_{k} B$ are similar, since $A O B \angle=A B E_{k} \angle$ and $B A O \angle=B A E_{k} \angle$ by Thm. 3. Therefore,

$$
\frac{\left|A E_{k}\right|}{|A B|}=\frac{|A B|}{|A O|} \text {, that is, } \frac{\left|A E_{k}\right|}{\mathrm{v}_{k}(b)}=\frac{\mathrm{v}_{k}(b)}{1} .
$$

Hence

$$
\left|A E_{k}\right|=\mathrm{v}_{k}(b)^{2} \text { and }\left|O E_{k}\right|=1-\mathrm{v}_{k}(b)^{2} .
$$

Let $C_{h}$ be the intersection of $B E_{k}$ and the covering line of the resulting body, and $C_{k}$ be the intersection of $A B$ line of the resulting body. Then, by the definition of collision ratio, see Def. 7 ,

$$
\left(b: e_{k}\right)_{k}=\frac{\left|C_{k} A\right|}{\left|B C_{k}\right|}, \quad \text { that is, } \quad\left|C_{k} A\right|=\left(b: e_{k}\right)_{k} \cdot\left|B C_{k}\right|,
$$


Figure 9: Proof of Thm. 11: Transformation of the Collision Ratio

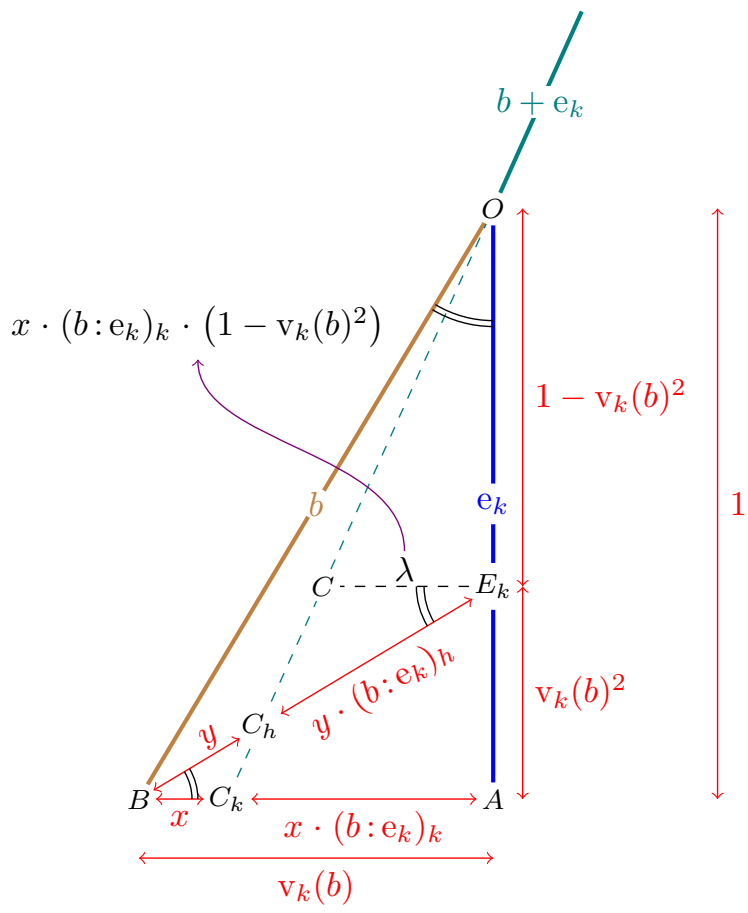

Since $\mathrm{w}_{k h}$ is an affine transformation by Thm. 3, it preserves ratios of Euclidean distances between points on a line. Therefore,

$$
\left(b: e_{k}\right)_{h}=\frac{\left|C_{h} E_{k}\right|}{\left|B C_{h}\right|}, \quad \text { that is, } \quad\left|C_{h} E_{k}\right|=\left(b: e_{k}\right)_{h} \cdot\left|B C_{h}\right| .
$$

Let $C$ be the coordinate point from the covering line of the resulting body which is simultaneous for $k$ with $E_{k}$.

Triangles $A O C_{k}$ and $E_{k} O C$ are similar, and the ratio of the similarity is $1-\mathrm{v}_{k}(b)^{2}$ by (8). Therefore, using (9), we have

$$
\left|C E_{k}\right|=\left|C_{k} A\right| \cdot\left(1-\mathrm{v}_{k}(b)^{2}\right)=\left(b: e_{k}\right)_{k} \cdot\left|B C_{k}\right| \cdot\left(1-\mathrm{v}_{k}(b)^{2}\right) .
$$

Now triangles $C_{h} E_{k} C$ and $C_{h} B C_{k}$ are also similar; therefore,

$$
\frac{\left|B C_{k}\right|}{\left|B C_{h}\right|}=\frac{\left|C E_{k}\right|}{\left|C_{h} E_{k}\right|} \text {. }
$$

Using (10) and (11) we can write the equation (12) in the following form:

$$
\frac{\left|B C_{k}\right|}{\left|B C_{h}\right|}=\frac{\left(b: e_{k}\right)_{k} \cdot\left|B C_{k}\right| \cdot\left(1-\mathrm{v}_{k}(b)^{2}\right)}{\left(b: e_{k}\right)_{h} \cdot\left|B C_{h}\right|},
$$

which simplifies to

$$
\left(b: \mathrm{e}_{k}\right)_{h}=\left(b: \mathrm{e}_{k}\right)_{k} \cdot\left(1-\mathrm{v}_{k}(b)^{2}\right) .
$$


By (13), we can change observer $h$ to $k$ in (7):

$$
\mathrm{m}_{0}(b)=\left(b: \mathrm{e}_{k}\right)_{k} \cdot\left(1-\mathrm{v}_{k}(b)^{2}\right) \cdot \frac{1}{\sqrt{1-\mathrm{v}_{k}(b)^{2}}}=\left(b: \mathrm{e}_{k}\right)_{k} \cdot \sqrt{1-\mathrm{v}_{k}(b)^{2}} .
$$

To turn $\left(b: \mathrm{e}_{k}\right)_{k}$ into mass, we only have to replace tandard-mass-equivalent $\mathrm{e}_{k}$ to the real mass-standard $\varepsilon$. Prop. 10 enables this step, so in $w^{\prime}$ we have

$$
\mathrm{m}_{0}(b)=\mathrm{m}_{k}(b) \cdot \sqrt{1-\mathrm{v}_{k}(b)^{2}} .
$$

And since this is an equation of two numbers, by AxMFrame (the invariance of mathematics), this equation holds also in the starting point $w$.

\section{Appendix}

\subsection{Poincaré Transformation Theorem}

Theorem 12.

MSpecRel $\vdash(\forall k, h \in \mathrm{IOb})$ " $\mathrm{w}_{k h}$ is a Poincaré transformation."

To prove that $\mathrm{w}_{k h}$ is a Poincaré transformation, it is enough to show that it takes lines of slope 1 to lines of slope 1, since there is an Alexandrov-Zeeman type theorem which works only with these premises, see [24]. To prove this lemma, let us introduce the following notation for the speed corresponding to coordinate points $\bar{x}$ and $\bar{y}$ :

$$
\mathrm{v}(\bar{x}, \bar{y}) \stackrel{\text { def. }}{=} \frac{\text { Space }(\bar{x}, \bar{y})}{\operatorname{Time}(\bar{x}, \bar{y})} .
$$

Lemma 13 (Light-line). Assume MSpecRel. Then every worldview transformation is a bijection taking lines of slope 1 to lines of slope 1 .

Proof. Worldview transformations are bijections by Prop. 2 .

Now we prove that worldview transformations take lines of slope 1 to lines of slope 1. By AxEField, $\mathrm{v}(\bar{x}, \bar{y})=\mathrm{v}(\bar{y}, \bar{z})=\mathrm{v}(\bar{z}, \bar{x})=1$ implies that $\bar{x}, \bar{y}$ and $\bar{z}$ are collinear. Therefore, to finish our proof, it is enough to derive the following formula:

$$
(\forall k, h \in \mathrm{IOb})(\forall \bar{x}, \bar{y})\left[\mathrm{v}(\bar{x}, \bar{y})=1 \rightarrow \mathrm{v}\left(\mathrm{w}_{k h}(\bar{x}), \mathrm{w}_{k h}(\bar{y})\right)=1\right] .
$$

Let $k$ and $h$ be arbitrary observers in a world $w$, and let $\bar{x}$ and $\bar{y}$ be coordinate points such that $\mathrm{v}(\bar{x}, \bar{y})=1$. By AxPhExp, in every $w$ world, there is an accessible world $w^{\prime}$ such that $w R w^{\prime}$ and in $w^{\prime}$ there is a light signal $p \in \operatorname{ev}_{k}(\bar{x}) \cap \mathrm{ev}_{k}(\bar{y})$ in $w^{\prime}$. By AxMFrame, $k$ still exists as an observer in $w^{\prime}$. So $p \in \operatorname{ev}_{k}\left(\mathrm{w}_{k h}(\bar{x})\right) \cap \mathrm{ev}_{k}\left(\mathrm{w}_{k h}(\bar{y})\right)$ by AxMEv. Consequently, by AxPhObs:

$$
\mathrm{v}\left(\mathrm{w}_{k h}(\bar{x}), \mathrm{w}_{k h}(\bar{y})\right)=1 ;
$$

and this is what we wanted to prove. 
Figure 10: The Symmetric Collision Theorem
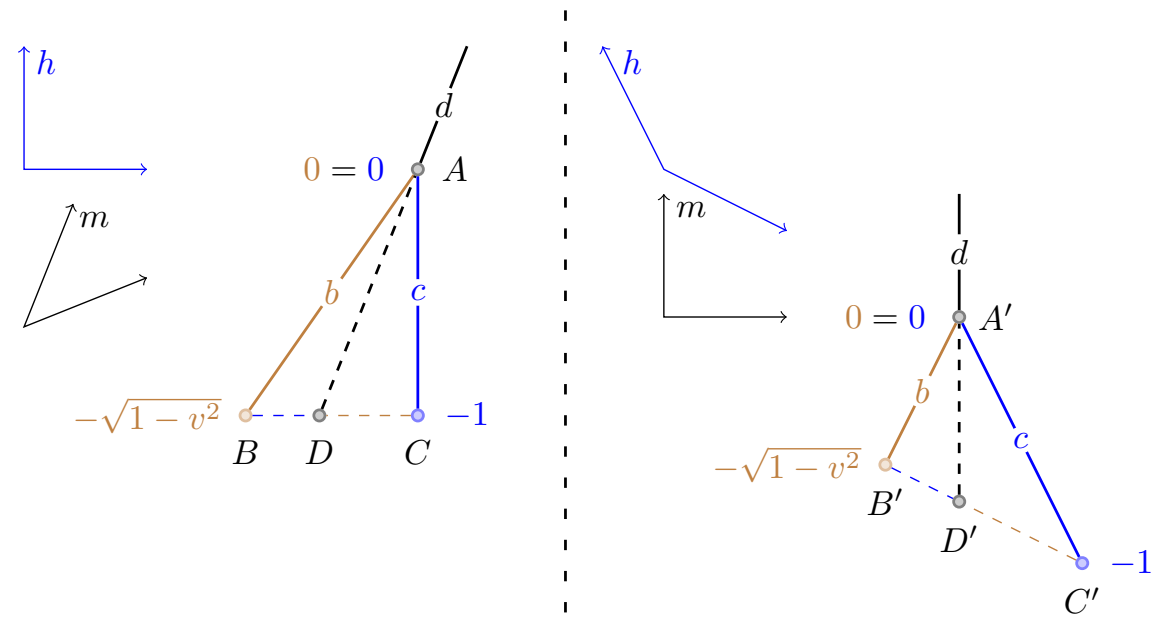

\subsection{Proof of Symmetric Collision Theorem}

Here we are going to prove Thm. 6 stating that:

\section{Theorem 14.}

MSpecRel $\vdash(\forall b, c \in \mathrm{OIB})(\forall k, h \in \mathrm{IOb})(\operatorname{SymColl}(b, c) \wedge$

$$
\left.\wedge \mathrm{v}_{k}(b)=0 \wedge \mathrm{v}_{h}(c)=0 \rightarrow(b: c)_{k}=\sqrt{1-\mathrm{v}_{k}(h)^{2}}\right) .
$$

Proof. Let $k$ and $h$ be observers and let $b$ and $c$ be ordinary bodies in a world $w$ such that $\mathrm{v}_{k}(b)=0, \mathrm{v}_{h}(c)=0$ and $\operatorname{SymColl}(b, c)$ holds for them. Since SymColl $(b, c)$ holds, there is an observer $m$ (the so-called median observer) in $w$ such that $\overline{\mathrm{v}}_{m}(b)+\overline{\mathrm{v}}_{m}(c)=\overline{0}$ and $(b: c)_{m}=1$. See Fig. 10.

The time dilation effect, i.e.,

$$
(\forall k, h \in \mathrm{IOb})\left(\forall \bar{x}, \bar{y} \in \operatorname{wline}_{k}(h)\right) \operatorname{Time}(\bar{x}, \bar{y})=\frac{\operatorname{Time}\left(\mathrm{w}_{k h}(\bar{x}), \mathrm{w}_{k h}(\bar{y})\right)}{\sqrt{1-\mathrm{v}_{k}(h)^{2}}},
$$

is a consequence of Thm. 3 , see [3, Thm. 2.4, (2)]:

We know from (15) that if the clocks of $k$ and $h$ show 0 at $A$, and the clock of $h$ shows -1 at $C$, then the clock of $k$ shows $-\sqrt{1-v^{2}}$ where $v$ is $\mathrm{v}_{h}(k)$.

We are interested in $(c: b)_{h}$, which is now:

$$
(c: b)_{h}=\frac{B D}{D C} .
$$

Since worldview transformation are affine transformations, this ratio is the same in the worldview of the median observer $m$, i.e.,

$$
\frac{B D}{D C}=\frac{B^{\prime} D^{\prime}}{D^{\prime} C^{\prime}}
$$


Since the worldline of $d$ is an angle bisector of the triangle $A^{\prime} B^{\prime} C^{\prime}$ in the worldview of $m$, by the angle bisector theorem,

$$
\frac{B^{\prime} D^{\prime}}{D^{\prime} C^{\prime}}=\frac{B^{\prime} A^{\prime}}{A^{\prime} C^{\prime}} .
$$

Since the clocks of $k$ and $h$ slow down with the same rate for the median observer $m$, we know that

$$
\frac{B^{\prime} A^{\prime}}{A^{\prime} C^{\prime}}=\sqrt{1-v^{2}}
$$

Since the collision was symmetric, $(b: c)_{k}=(c: b)_{h}$. Therefore, from (16), (17), (18) and (19) we have

$$
(c: b)_{k}=\sqrt{1-v^{2}},
$$

and this is what we wanted to prove.

\subsection{Consistency and Independence}

To prove the consistency of MSpecRelDyn, it is enough to construct a model for it. It is easy to construct a model of MSpecRelDyn - if we choose $\mathrm{IOb}^{\mathfrak{M}}$ and $\mathrm{W}^{\mathfrak{M}}$ to be empty, then almost all axioms will be satisfied, since all of them has the form " $\forall k \in \mathrm{IOb}) . .$. ". The only exception is AxMFrame, since this requires the reflexivity of the alternative relation, the rigidity of mathematics, etc., but it is easy to satisfy these statements by choosing the set of possible worlds to be a singleton $\{w\}$ such that $w R w$.

However, we would not only like to prove that that MSpecReIDyn is consistent but also that it has some complex models, models where there are several observers moving relative to one another and measuring ordinary inertial bodies, e.g., where we see something similar to what we had in mind during the proofs of Section 4. This type of model construction is too complex to be included in detail in this paper. However, in this section, we give a sketch of such a construction.

The main difficulty in the construction of such a model is the following: If two ordinary bodies, say $b$ and $c$ collide, then every observer has to measure the two bodies and the resulting body $b \oplus c$ directly, pseudo-directly or indirectly. There are plenty of other measurements we have to include because, for example, in the successor world, where the pseudo-direct measurement of $b$ is done by $\mathrm{e}_{k}$, there will be an ordinary body $b \oplus \mathrm{e}_{k}$, which again, has to be measured directly, pseudo-directly, indirectly, and so on and so forth. So building any model $\mathfrak{M}$ in which MSpecRelDyn is true (i.e., true in every world of $\mathfrak{M}$ ), and in which $\exists 2 \mathrm{IOb}$ and $(\exists b) \operatorname{OIB}(b)$ is satisfied (i.e., true in some world of $\mathfrak{M}$ ) involves an infinite process of measuring.

Another difficulty is that by $\exists 2 \mathrm{IOb}$ two observers sooner or later will compare their equivalents by AxPDirComp, which postulates the existence of a medianobserver as well. So the cardinality of observers cannot be 2 or any finite number.

From now on, to describe a complex model $\mathfrak{M}$, we take the perspective of an observer $M$. To guarantee the truth of $\exists 2 \mathrm{IOb}$ and the existence of medians (needed for AxPDirComp), we include infinitely many ${ }^{23}$ observers to meet with

\footnotetext{
${ }^{23}$ We take infinite number of observers only to simplify the construction. It is also possible to construct a model where there are only finitely many observers in every world.
} 
Figure 11: A part of the model: measurements concerning two equivalents $a$ and $b$ of observers $A, B$ having opposite velocity according to $M$

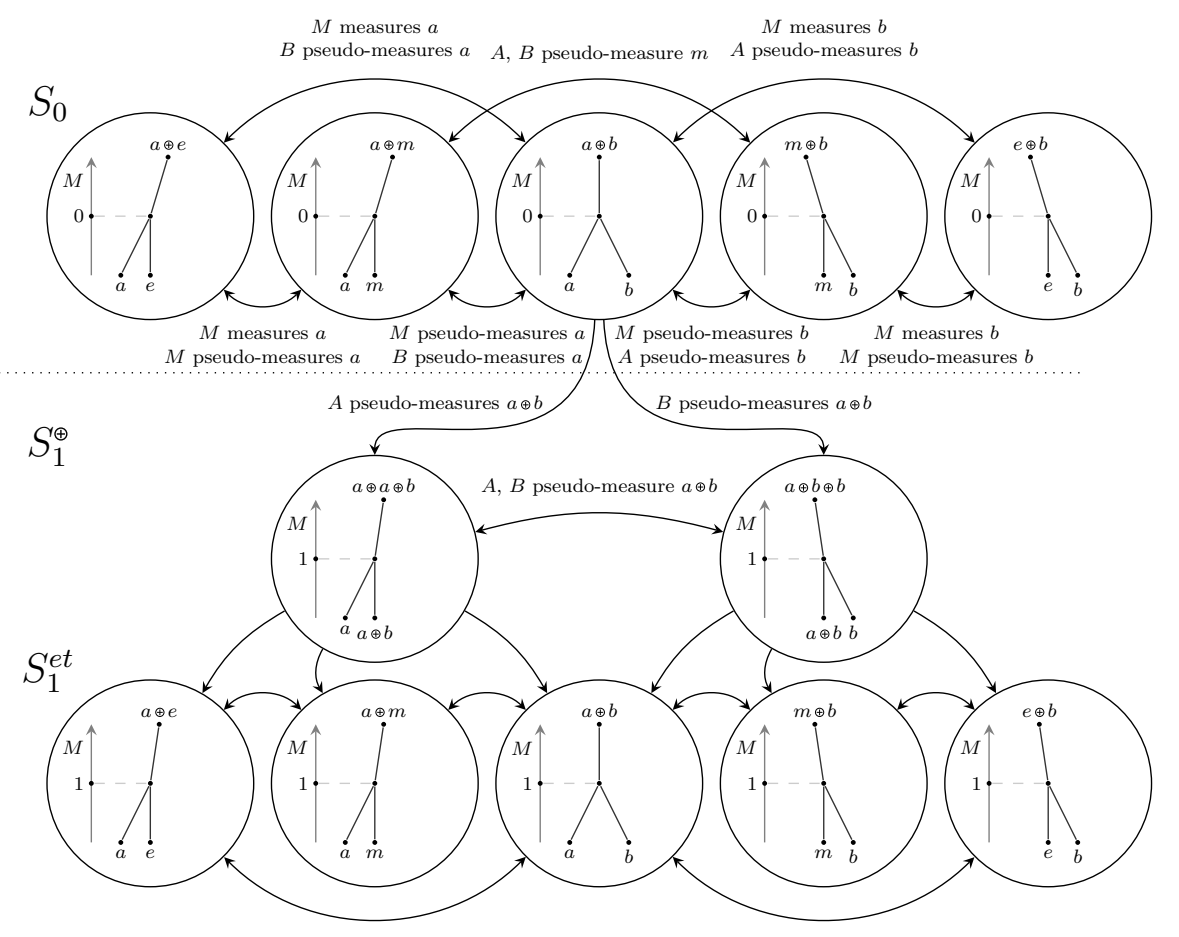

$M$ in its origin in all worlds (where $M$ exists) such that for every possible velocity in the interval $[-0.5,0.5]$, there will be an observer in the $t x$-plane having that velocity. There will be a set of possible worlds, $S_{0}$, where these observers compare their mass-standard-equivalents and measure the other's equivalent directly in the origin at time 0 according to $M$. Every such comparison and direct measurement will result a body, which again has to be measured; these measurements will happen 1 second later according to $M$, and these worlds will constitute the set $S_{1}^{\oplus}$. Note that mass-standard-equivalents of $S_{1}^{\oplus}$ have different worldlines than they had in $S_{0}$ since their worldlines end at the time 1 instead of 0 . So there will be a set $S_{1}^{\text {et }}$ very similar to $S_{0}$, where the observers compare their mass-standard-equivalents 1 second later. The construction continues in the same way with $S_{2}^{\oplus}, S_{2}^{e t}, S_{3}^{\oplus}, S_{3}^{e t} \ldots$ into infinity. A part of that model, measurements concerning two equivalents $a$ and $b$ of observers $A, B$ having opposite velocity according $M$, is illustrated on Fig. 11. This figure shows also how are the indirect measurements fulfilled in that model: $a \oplus b$ is measured in the central world of the figure indirectly through worlds having the resulting body $a \oplus a \oplus b$ and $a \oplus e$ (the latter is from $S_{1}^{e t}$ ).

This construction will satisfy all the axioms of MSpecRelDyn except the light signal-sending axiom AxPhExp. To make this axiom true in every world of our model, it is enough to extend this construction with only one world, say $p h$, which is an alternative of every world of the model, and which realizes every 
possible photon needed by any world. ${ }^{24}$ This means that $p h$ will be a classical model of SpecRel. For such a construction, see [3, Corollary 11.12, especially pp. 643-644.].

This construction can be carried out even in a way such that the conservation of mass and linear moment fails in the resulting model. This construction shows that the Mass Increase Theorem can be proved without the usual conservation postulates, which is a result similar to that of [4]. ${ }^{25}$ However, MSpecRelDyn has models (different from the above construction) where even the key axiom of [4], AxCenter, the conservation of centerline of mass, is refutable. ${ }^{26}$

Independence. This model $\mathfrak{M}$ can easily be modified to show the independence of several axioms of MSpecRelDyn. For example, if we remove a pair of worlds $\left\langle w, w^{\prime}\right\rangle$ from the alternative relation where $w^{\prime}$ is a world where the mass-standard exists, then we can falsify AxDir while the other axioms remain true, i.e., AxDir is independent from the rest axioms of MSpecRelDyn. If we do the same with a $w^{\prime}$ where instead of the mass-standard, one of its equivalents exists, we have the independence of AxPDirComp. To show that AxCollRel is independent, it is enough to copy an arbitrary world of $\mathfrak{M}$ which sees the same alternatives, but which is seen by no one. If, in this copied world, we alter the speed of one body, we can violate AxCollRel while the other axioms remain true.

The independence of AxIndir from the rest of the axioms of MSpecRelDyn can be showed by a one-world model where there is only one observer and only one resting ordinary inertial body resting to that observer. However, in the light of our motivation of $\mathfrak{M}$, it is worth to examine the independence of AxIndir in the axiom system MSpecRelDyn $\cup\{\exists 2 \mathrm{IOb}\}$ as well. The existence of indirect measurements is a consequence of MSpecRelDyn $\cup\{\exists 2 I O b\}$, the idea is used in the proof of the Mass Increase Theorem (Thm. 11). However, the independence of the uniqueness of the results of indirect measurements is a question for further research. Our conjecture is that it is independent.

It is also a question for further research whether the last axiom of MSpecRelDyn, AxEqSym is independent from the rest of MSpecRelDyn or not; our conjecture is that it is. However, a model which would be capable of showing this must be entirely different from the above outlined $\mathfrak{M}^{27}$

\section{Concluding Remarks}

We have seen that the act of thought experimentation is a formalizable notion. The formal counterparts of thought experiments are model transformations of classical models. Modal logic express a large amount of these transformations. If we stay at the level of first-order logic, we still have a strong completeness theorem which is essential in foundational axiomatic approaches. With the aid

\footnotetext{
${ }^{24}$ Again, at the cost of simplicity, it is possible to construct a model where there are only finitely many photons in every world.

${ }^{25}$ The key idea here is that we can adjust the worldlines in all worlds in a way that all collisions of the model became symmetric. This causes that all body became an mass-standardequivalent in the model, even the results of the collision of mass-standard-equivalents, which is a violation of the law of the conservation of mass.

${ }^{26}$ For the key idea, see footnote 22 .

${ }^{27}$ We suspect that the underlying directed graph drawn by the alternative relation of a model capable of showing the independence of AxEqSym must be very close to a tree.
} 
of first-order modal logic, we axiomatized relativistic Kinematics and Dynamics with formal thought experiments.

An advantage of this approach is that we can distinguish between actual and theoretical (or possible) objects. The philosophical importance of this is that the ontological statuses of actual and potential objects are clearly different. Theoretical objects are just non-existing objects that we need to prove our statements. For example, the possible photons postulated by AxPhExp are needed to prove that the worldview transformations are Poincare transformations. The transmitting bodies of AxIndir, the mass-standard and its equivalents of AxDir and AxPDirComp, respectively, are needed to give a well-defined concept of relativistic mass based on only kinematical terms.

Using this definition and AxEqSym, we proved the Mass Increase Formula without using the conservation postulates about linear momentum, mass, or even the centerline of relativistic mass (the key axiom of [4]). This result suggests that the presence of formal thought experiments make it possible to 'dig deeper' in the foundations of relativity theories.

\section{References}

[1] H. Andréka, J. X. Madarász, and I. Németi, with contributions from: A. Andai, G. Sági, I. Sain, and Cs. Tóke. On the logical structure of relativity theories. Research report, Alfréd Rényi Institute of Mathematics, Hungar. Acad. Sci., Budapest, 2002. http://www.math-inst.hu/pub/algebraiclogic/Contents.html.

[2] H. Andréka, J. X. Madarász, and I. Németi. Logical analysis of relativity theories. In V. F. Hendricks et al., editors, First-Order Logic Revisited, pages 7-36. Logos Verlag, Berlin, 2004.

[3] H. Andréka, J. X. Madarász, and I. Németi. Logic of spacetime and relativity theory. In M. Aiello, I. Pratt-Hartmann, and J. van Benthem, editors, Handbook of spatial logics, pages 607-711. Springer-Verlag, Dordrecht, 2007.

[4] H. Andréka, J. X. Madarász, I. Németi, and G. Székely. Axiomatizing relativistic dynamics without conservation postulates. Studia Logica, 89(2):163-186, 2008.

[5] H. Andréka, J. X. Madarász, I. Németi, and G. Székely. On Logical Analysis of Relativity Theories. Hungarian Philosophical Review, 2010/4:p.204-222, 2011. arXiv: 1105.0885 .

[6] H. Andréka, J. X. Madarász, I. Németi, and G. Székely. A logic road from special relativity to general relativity. Synthese, 186(3):633-649, 2012.

[7] J. Ax. The elementary foundations of spacetime. Found. Phys., 8(7-8):507$546,1978$.

[8] T. Benda. A Formal Construction of the Spacetime Manifold. J. Phil. Logic, 37(5):441-478, 2008. 
[9] P. Blackburn, M. de Rijke, and Y. Venema. Modal Logic. Cambridge Tracts in Theoretical Computer Science. Cambridge University Press, 2002.

[10] C. C. Chang and H. J. Keisler. Model theory. North-Holland Publishing Co., Amsterdam, 1990

[11] G. Corsi. A Unified Completeness Theorem for Quantified Modal Logics. The Journal of Symbolic Logic, 67(4), 2002.

[12] A. Einstein. Zur Elektrodynamik bewegter Körper. Annalen der Physik, 17:891-921, 1905.

[13] K. Fine. The Permutation principle in quantificational logic. Journal of Philosophical Logic, 12:33-37, 1983.

[14] R. Goldblatt. Diodorean Modality in Minkowski Spacetime. Studia Logica, (39):219-236, 1980.

[15] R. Goldblatt. Orthogonality and spacetime geometry. Springer-Verlag, New York, 1987.

[16] R. Goldblatt. Quantifiers, Propositions and Identity: Admissible Semantics for Quantified Modal and Substructural Logics. Lecture Notes in Logic. Cambridge University Press, 2011.

[17] A. Hazen. Expressive completeness in modal languages. Journal of Philosophical Logic, 5:25-46., 1976.

[18] R. Hirsch. Modal Logic and Relativity. Hungarian Philosophical Review, 54(4):223-230, 2010.

[19] H. T. Hodes. Some theorems on the expressive limitations of modal languages. Journal of Philosophical Logic, 13(1):13-26, 1984.

[20] B. Hoffman. A logical treatment of special relativity, with and without faster-thanlight observers, 2013. BA Thesis.

[21] M. Jammer. Concepts of Mass in Contemporary Physics and Philosophy. Princeton University Press, 2000.

[22] J. X. Madarász. Logic and Relativity (in the light of definability theory). $\mathrm{PhD}$ thesis, Eötvös Loránd Univ., Budapest, 2002. http://www.mathinst.hu/pub/algebraic-logic/Contents.html.

[23] J. X. Madarász and G. Székely. Comparing Relativistic and Newtonian Dynamics in First-Order Logic. In A. Máté, M. Rédei, and F. Stadler, editors, Der Wiener Kreis in Ungarn / The Vienna Circle in Hungary, volume 16 of Veröffentlichungen des Instituts Wiener Kreis, pages 155179. Springer Vienna, 2011.

[24] J. X. Madarász and G. Székely. Special Relativity over the Field of Rational Numbers. International Journal of Theoretical Physics, pages 1-13, 2013.

[25] J. X. Madarász and G. Székely. The existence of superluminal particles is consistent with relativistic dynamics. Journal of Applied Logic, 2014. (in press) 
[26] A. Molnár. On the Notion of Possibility in Relativity Theory, September $2012 . \quad$ Conference talk: http://www.renyi.hu/conferences/nemeti70/LR12Talks/molnar.pdf.

[27] A. Molnár. Lehetségesség a fizikában. Elpis, 7(1):73-103, June 2013.

[28] V. Pambuccian. Alexandrov-Zeeman type theorems expressed in terms of definability. Aequationes Math., 74(3):249-261, 2007.

[29] I. Shapirovsky and V. Shehtman. Modal Logics of Regions and Minkowski Spacetime. Journal of Logic and Computation, 15(4):559-574, 2005.

[30] V. B. Shehtman. Modal logics of domains on the real plane. Studia Logica, 42(1):63-80, 1983.

[31] G. Székely. First-Order Logic Investigation of Relativity Theory with an Emphasis on Accelerated Observers. PhD thesis, Eötvös Loránd Univ., Budapest, 2009.

[32] G. Székely. The existence of superluminal particles is consistent with the kinematics of Einstein's special theory of relativity. Reports on Mathematical Physics, 72(2):133-152, 2013. 